\title{
The observed velocity distribution of young pulsars
}

\author{
Frank Verbunt, Andrei Igoshev, and Eric Cator
}

\author{
Institute of Mathematics, Astrophysics and Particle Physics, Radboud University Nijmegen, PO Box 9010, 6500 GL Nijmegen, \\ The Netherlands \\ e-mail: [F.Verbunt;A.Igoshev]@astro.ru.nl; E.Cator@science.ru.nl
}

Received 6 July 2017 / Accepted 29 September 2017

\begin{abstract}
We argue that comparison with observations of theoretical models for the velocity distribution of pulsars must be done directly with the observed quantities, that is parallax and the two components of proper motion. We have developed a formalism to do so, and applied it to pulsars with accurate VLBI measurements. For computational convenience, we model the data with Maxwellians. We find that a distribution with two Maxwellians improves significantly on a single Maxwellian. The "mixed" model takes into account that pulsars move away from their place of birth, a narrow region around the Galactic plane. The best model has $42 \%$ of the pulsars in a Maxwellian with average velocity $\sigma \sqrt{8 / \pi}=120 \mathrm{~km} \mathrm{~s}^{-1}$, and $58 \%$ in a Maxwellian with average velocity $540 \mathrm{~km} \mathrm{~s}^{-1}$. About $5 \%$ of the pulsars has a velocity at birth less than $60 \mathrm{~km} \mathrm{~s}^{-1}$. For the youngest pulsars $\left(\tau_{\mathrm{c}}<10 \mathrm{Myr}\right)$, these numbers are $32 \%$ with $130 \mathrm{~km} \mathrm{~s}{ }^{-1}$, $68 \%$ with $520 \mathrm{~km} \mathrm{~s}^{-1}$, and $3 \%$, with appreciable uncertainties. Our analysis shows that the velocity distribution is wider than can be described with a single Maxwellian; it does not prove that two Maxwellians provide a better description than other wide models.
\end{abstract}

Key words. stars: neutron - pulsars: general - methods: statistical

\section{Introduction}

The study of the velocities of pulsars is interesting on its own account, as a pointer to the formation process of a neutron star, but also has ramifications beyond this. In particular, some neutron stars are found in binaries and in globular clusters, as accreting $\mathrm{X}$-ray sources or as pulsars. These neutron stars were born with velocities less than the escape velocity from the binary or from the cluster.

Neutron stars that have the same velocities as their progenitors, move with the rotation of the galaxy, with small velocities with respect to the local standard of rest (LSR), unless their progenitor is a member of a close binary or a runaway star. To investigate the velocities that neutron star acquires at birth in addition to the progenitor velocity, one therefore investigates their velocity $v$ with respect to the LSR.

This investigation is complicated for pulsars with large velocities as these are affected by an acceleration in the Galactic potential that varies between their place of birth and their current location, and because their current LSR differs from the LSR at their place of birth. Thus the current $v$ of a pulsar differs from the $v$ at birth. If the age and full space velocities were known, we could solve this complication by integrating the pulsar orbit back in time, but proper motion studies only provide two of the three velocity components, and ages of pulsars are usually uncertain. By limiting the study to young pulsars, one may reduce the effect of these complications. As well described by Brisken et al. (2003a, in particular Sect. 5.1), correlations between spin-axis and velocity, between luminosity and velocity, and/or between velocity and distance to the Galactic plane, among others, introduce selection effects in the observations. Such selection effects can only be corrected for in a full population study. Even so, determining the observed $v$ distribution is a useful step toward a full population study, and various efforts have been published (see Table 5).
Arzoumanian et al. (2002) compare synthesized model populations with the observed periods, period derivatives, dispersion measures, fluxes, and the absolute values of Galactic latitudes and of proper motions. They conclude that the velocity distribution of pulsars is bimodal, with a low-velocity and a highvelocity component.

Brisken et al. (2003a) investigate the velocity component $v_{l}$ in the direction of Galactic longitude. Their study is based on interferometric proper motion measurements (mostly their own). For each pulsar, they compute a probability distribution $P(D)$ for the distance $D$ (based on the parallax or on the dispersion measure DM, allowing for the limited accuracy in converting DM to $D)$ and combine this with the probability function $P\left(\mu_{l}\right)$ for the proper motion $\mu_{l}$ (allowing for measurement uncertainty) to compute the probability distribution $P\left(v_{l}\right)$. The set of $P\left(v_{l}\right)$ is fitted with a model in which this distribution is described by two zero-centred Gaussian distributions, representing a slow and a fast component.

Hobbs et al. (2005) construct velocity distributions $P\left(v_{1 \mathrm{D}}\right)$ where $v_{1 \mathrm{D}}$ is either $v_{l}$ or $v_{b}$ and $P\left(v_{2 \mathrm{D}}\right)$ where $v_{2 \mathrm{D}} \equiv \sqrt{v_{l}^{2}+v_{b}{ }^{2}}$ for a larger sample of pulsars, including measurements based on timing. $v_{b}$ is the velocity component in the direction of latitude. Hobbs et al. assume that these observed $v_{1 \mathrm{D}}$ and $v_{2 \mathrm{D}}$ distributions are projections of an isotropic velocity distribution $P(v)$, and then reconstruct $P(v)$ by using a clean algorithm to deconvolve $P\left(v_{1 \mathrm{D}}\right)$ and $P\left(v_{2 \mathrm{D}}\right)$. The advantage of this method is that it is non-parametric, that is it does not assume a prescribed form for $P(v)$. The reconstructed form turns out to be well described by a Maxwellian, with $\sigma=265 \mathrm{~km} \mathrm{~s}^{-1}$.

Faucher-Giguère \& Kaspi (2006) extend the method of Brisken et al. (2003a) in two ways. First they consider a variety of models for the distribution of $v_{l}$, and second they extend the maximum-likelihood model with a Bayesian analysis of probability ratios for the comparison of different models. 
Whereas these studies agree that the space (that is $3 \mathrm{D}$ ) velocities of neutron stars are high, averaging as much as $450 \mathrm{~km} \mathrm{~s}^{-1}$, they differ on the fraction of low-velocity neutron stars. Hobbs et al. (2005) argue that the low-velocity tail of the pulsar velocity distribution is due to projection effects, and that very few pulsars have space velocities below $60 \mathrm{~km} \mathrm{~s}^{-1}$. (For a Maxwellian with $\sigma=265 \mathrm{~km} \mathrm{~s}^{-1}$ the fraction is 0.003 .) In the acceptable models discussed by Faucher-Giguère \& Kaspi (2006) the derived fraction of pulsars with space velocities less than $60 \mathrm{~km} \mathrm{~s}^{-1}$ varies from 0.012 (for a two-component Gaussian) to 0.135 (for the Paczyński distribution).

One reason for us to make a new study of the pulsar velocities is to resolve the differences between the predicted numbers of low-velocity pulsars in these recent studies. Among nine very accurate pulsar velocities $v_{\perp}\left(=\sqrt{v_{l}^{2}+v_{b}^{2}}\right)$ listed by Brisken et al. (2002, Table 5), two are smaller than $40 \mathrm{~km} \mathrm{~s}^{-1}$. The probability of finding two such low- $v_{\perp}$ pulsars in a sample of nine is 0.004 for an isotropic Maxwellian with $\sigma=265 \mathrm{~km} \mathrm{~s}^{-1}$. This suggests that the pulsar velocities may be overestimated by Hobbs et al. (2005).

A second reason for a new study is the development by Verbiest et al. (2012), of a Bayesian method to combine different distance indicators into a single probability distribution $P(D)$ for each pulsar. The main distance indicator is the parallax, where the Lutz-Kelker (1973) effect is taken into account, with the Galactic pulsar distribution as a prior. For the study of pulsar velocities we correct some errors in the equations given by Verbiest et al. (2012) for use of the parallax (see Igoshev et al. 2016 and Bailer-Jones 2015), and add the measurements of the proper motions. The third and final reason for our new study of pulsar velocities is the increased number of accurately measured proper motions and parallaxes (see Table 2).

In Sect. 2 we describe the master list of observed proper motions that we use in our study. We describe the ingredients of the likelihood function for pulsars and their use in determining the parameters of the velocity distribution in Sect.3, and apply these to various models: a single isotropic Maxwellian in Sect. 4, the sum of two isotropic Maxwellians in Sect. 5, and a mixture of one or two isotropic and semi-isotropic Maxwellians in Sect. 7. (In the semi-isotropic Maxwellian distribution velocities towards the Galactic plane are excluded, as explained in Sect. 6.) We use Maxwellians for computational convenience: an isotropic Maxwellian may be decomposed in three independent Gaussians in any three mutually perpendicular directions, and as a result a large part of the calculations can be done analytically. The use of Maxwellians futhermore enables direct comparisons with previous papers, most of which also use Maxwellians. We leave the rather more complicated study of other models to future work.

Before we proceed, we describe the notation we use: we differentiate between the actual (and generally unknown) properties of the pulsar, and the measured (or nominal) values, by indicating the latter with a prime $\left({ }^{\prime}\right)$. The actual proper motion is the sum of three components: one due to the peculiar velocity of the pulsar, one due to the difference between the local standards of rest of the pulsar and of the Sun, and one due to the peculiar motion of the Sun (Eqs. (6)-(9)). The measured parallax and proper motion differs from the actual values due to measurement errors (Eqs. (1)-(3)), and may be skewed due non-uniform distributions of positions and velocities (Fig. 2). For convenience, our notation is summarized in Table 1.
Table 1. Notation used in this paper.

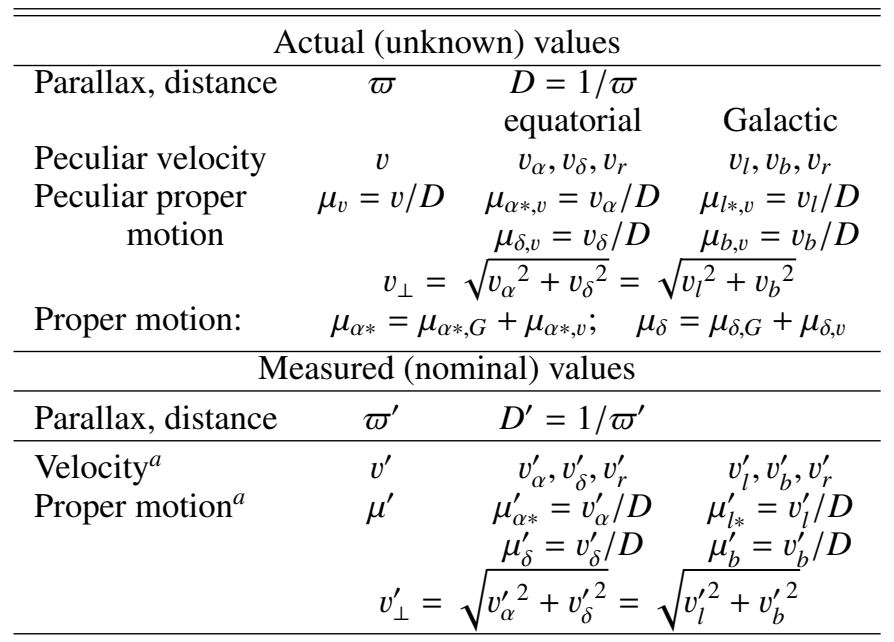

Notes. ${ }^{(a)}$ The measured values differ from the actual values not only due to measurement error, but also due to correction for Galactic rotation.

\section{Data}

To obtain a master list of pulsars with measured proper motions, we start by collating articles with proper motion measurements. The ATNF Catalogue, version $1.54^{1}$ (Manchester et al. 2005), was very helpful in this.

Brisken et al. (2000) note that VLBI measurements of proper motions need to be corrected for ionospheric refraction. We therefore do not use articles with proper motions from VLBI published before 2000. To select first-born, single pulsars we reject recycled pulsars (that is those with $\dot{P}<5 \times 10^{-18} \mathrm{~s} \mathrm{~s}^{-1}$ ), pulsars in binaries, and pulsars in globular clusters.

In this first application of our new method we prefer to use relatively accurate measurements. We therefore omit pulsars with distances determined only from dispersion measures, and pulsars with proper motions determined from pulse timing. In both cases, the errors are at least an order of magnitude larger than the errors obtained with VLBI, and often only correspond to (upper or lower) limits. Distances from dispersion measures have uncertainties dominated by systematic effects, with highly non-Gaussian distributions. (For pulsars distances and dispersion measures, see for example Yao et al. 2017; for proper motions from timing, see Hobbs et al. 2004.) We also omit proper motions of pulsars derived from displacements in X-ray or optical images, which are relative to other objects in the field of view. The conversion to absolute proper motions in the International celestial reference system (ICRS) adds significantly to the error.

This leaves us with the VLBI measurements of the articles listed in Table 2. Although the measurement of the proper motion components are not independent of each other, the covariance value is only provided by Brisken et al. (2003a) who give no parallax values. We therefore ignore covariances between $\mu_{\alpha *}^{\prime}$ and $\mu_{\delta}^{\prime}$. In the majority of the measurements, the errors are symmetric, and where asymmetric, the difference is small. We simplify our analysis by taking the largest error when errors are asymmetric. (Test calculations in which the smallest error is taken give the same results.)

\footnotetext{
1 www.atnf.csiro.au/research/pulsar/psrcat
} 

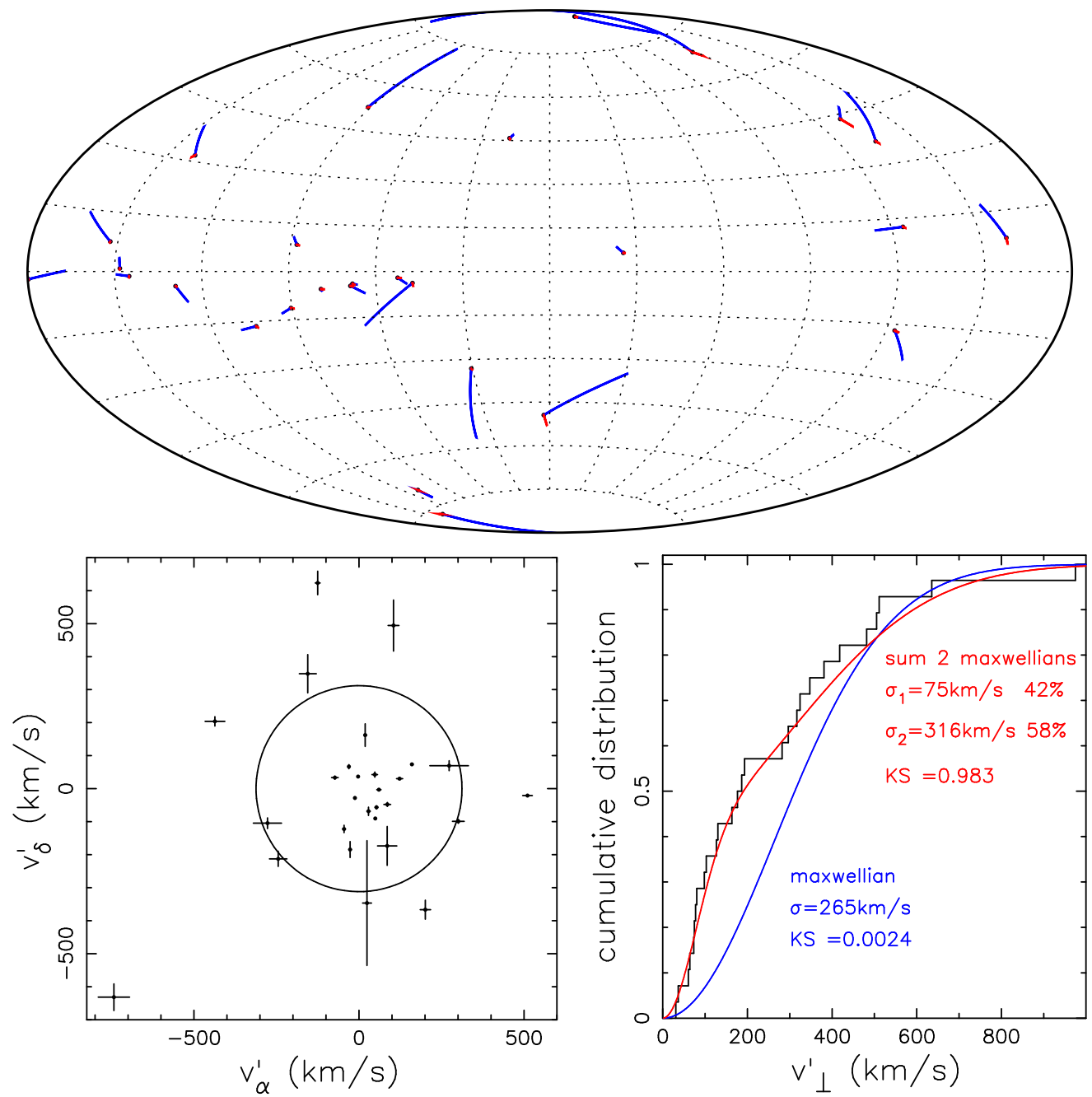

Fig. 1. Illustrations of data from our master list of pulsars Table D.1. Top: celestial distribution in Galactic coordinates. The blue lines show the observed proper motion $\mu^{\prime}$ and the red lines the correction due to Galactic rotation (for nominal distance $D^{\prime}$ ), in 0.5 Myr. Below left: nominal velocities in the celestial plane. The circle indicates the median value for $v_{\perp}$ for the projection of a Maxwellian: $\sqrt{2 \ln 2} \sigma$, for $\sigma=265 \mathrm{~km} \mathrm{~s}^{-1}$. Below right: cumulative distributions of the observed $v_{\perp}^{\prime}$, and of $v_{\perp}$, blue: according to Hobbs et al. (2005), red: according to our best solution, with the $p$-value according to a one-sided Kolmogorov-Smirnov test that the observed distribution is drawn from the theoretical one.

Table 2. Sources for proper motions in our master list.

\begin{tabular}{r|lrrr}
\hline \hline$S$ & \multicolumn{1}{c}{ Source } & & $N$ & $n$ \\
\hline 1 & Brisken et al. (2002) & Table 4 & 6 & 2 \\
2 & Brisken et al. (2003b) & Table 3 & 1 & 1 \\
3 & Chatterjee et al. (2001) & Table 2 & 1 & 1 \\
4 & Chatterjee et al. (2004) & Table 1 & 1 & 1 \\
5 & Deller et al. (2009) & Table 3 & 4 & 2 \\
6 & Chatterjee et al. (2009) & Table 2 & 12 & 9 \\
7 & Kirsten et al. (2015) & Table 5 & 3 & 3 \\
& & Total: & 28 & 19 \\
\hline
\end{tabular}

Notes. $S$ source indicator, $N$ number of entries used (isolated pulsars with parallax measurements), $n$ with age less than 10 Myr. Later measurements may replace earlier ones; the source actually used is indicated in Table D.1.

The resulting master list of observed proper motions in equatorial coordinates is given in Table D.1. The proper motions in this table are the observed proper motions $\mu_{\alpha *}^{\prime}$ and $\mu_{\delta}^{\prime}$, not corrected for Galactic rotation and peculiar solar velocity. The celestial distribution, measured proper motions and nominal velocities of the pulsars in our master list are illustrated in Fig.1.
From the top figure we learn that the correction for Galactic motion in general is small. The lower figures add to our suspicion that a single Maxwellian with $\sigma=265 \mathrm{~km} \mathrm{~s}^{-1}$ seriously underestimates the number of pulsars with low velocities.

\section{Ingredients}

\subsection{Probabilities}

To determine the pulsar velocity distribution we use the measured values of the parallax $\varpi^{\prime}$ and of the two components of the proper motion $\mu_{\alpha *}^{\prime}$ and $\mu_{\delta}^{\prime}$. The conditional probabilities of obtaining these measured values when the actual values are $\varpi=1 / D, \mu_{\alpha *}$ and $\mu_{\delta}$ can be written separately as

$$
\begin{aligned}
& g_{D}\left(\varpi^{\prime} \mid D\right) \Delta \varpi^{\prime}=\frac{1}{\sqrt{2 \pi} \sigma_{\varpi}} \exp \left[-\frac{\left(1 / D-\varpi^{\prime}\right)^{2}}{2 \sigma_{\varpi}^{2}}\right] \Delta \varpi^{\prime}, \\
& g_{\alpha}\left(\mu_{\alpha *}^{\prime} \mid \mu_{\alpha *}\right) \Delta \mu_{\alpha *}^{\prime}=\frac{1}{\sqrt{2 \pi} \sigma_{\alpha}} \exp \left[-\frac{\left(\mu_{\alpha *}-\mu_{\alpha *}^{\prime}\right)^{2}}{2 \sigma_{\alpha}^{2}}\right] \Delta \mu_{\alpha *}^{\prime}, \\
& g_{\delta}\left(\mu_{\delta}^{\prime} \mid \mu_{\delta}\right) \Delta \mu_{\delta}^{\prime}=\frac{1}{\sqrt{2 \pi} \sigma_{\delta}} \exp \left[-\frac{\left(\mu_{\delta}-\mu_{\delta}^{\prime}\right)^{2}}{2 \sigma_{\delta}^{2}}\right] \Delta \mu_{\delta}^{\prime}
\end{aligned}
$$


where $\sigma_{\varpi}, \sigma_{\alpha}$ and $\sigma_{\delta}$ are the measurement errors for the parallax and for the two components of the proper motion, respectively. To obtain the joint probability of the measured and actual values, these equations must be complemented with the equations indicating the probability density functions of the actual distance and proper motion.

The probability density $f_{D}(D)$ of the distance $D$ of the pulsar to the Earth for a galactocentric pulsar distribution is given by Verbiest et al. (2012). In the notation of Igoshev et al. (2016):

$f_{D}(D) \propto D^{2} R^{1.9} \exp \left[-\frac{|z(D, b)|}{h}-\frac{R(D, l, b)}{H}\right] \equiv D^{2} \mathcal{F}(D)$,

with

$z=D \sin b ;$ and $R=\sqrt{R_{0}^{2}+(D \cos b)^{2}-2 D \cos b R_{0} \cos l}$,

where $R$ and $R_{0}$ are the galactocentric distance of the pulsar and the Sun, respectively, projected on the Galactic plane. Through $\mathcal{F}(D)$ also $f_{D}(D)$ is a function of Galactic coordinates $l, b$.

\subsection{Proper motions}

The proper motion of a pulsar $\mu_{\alpha^{*}}, \mu_{\delta}$ is the sum of the proper motion of its standard of rest with respect to the Sun $\mu_{\alpha *, G}, \mu_{\delta, G}$ and the proper motion caused by its velocity with respect to its local standard of rest $\mu_{\alpha *, v}, \mu_{\delta, v}$ :

$\mu_{\alpha *}=\mu_{\alpha *, G}+\mu_{\alpha *, v} ; \quad \mu_{\delta}=\mu_{\delta, G}+\mu_{\delta, v}$.

The derivation of $\mu_{\alpha *, G}$ and $\mu_{\delta, G}$ is described in Appendices A and $\mathrm{B}$. The velocity of the local standard of rest is assumed to be the Galactic rotation velocity, $v_{R}\left(R_{0}\right)$ for the Sun and $v_{R}(R)$ for the pulsar. The peculiar velocity of the Sun is $[U, V, W]$, where the components are respectively in the direction from the Sun towards the Galactic centre, in the direction of the Galactic rotation, and perpendicular to the Galactic plane. In Galactic coordinates

$D \mu_{l *, G}=U \sin l-\left[V+v_{R}\left(R_{0}\right)\right] \cos l+v_{R}(R) \cos (\theta+l)$,

and

$$
\begin{aligned}
D \mu_{b, G}= & {\left[U \cos l+\left[V+v_{R}\left(R_{0}\right)\right] \sin l-v_{R}(R) \sin (\theta+l)\right] \sin b } \\
& -W \cos b .
\end{aligned}
$$

The angle $(\theta+l)$ is computed from:

$\tan (\theta+l)=\frac{R_{0} \sin l}{R_{0} \cos l-D \cos b}$.

The values for $[U, V, W], v_{R}$ and $R_{0}$ that we use are listed in Table 3. To compare velocities expressed in $\mathrm{km} \mathrm{s}^{-1}$ with proper motions expressed in mas/yr, we use the conversion

$v\left(\mathrm{~km} \mathrm{~s}^{-1}\right)=4.74 \mu(\mathrm{mas} / \mathrm{yr}) D(\mathrm{kpc})$.

The pair $\mu_{l *, G}, \mu_{b, G}$, is converted to the pair in equatorial coordinates $\mu_{\alpha *, G}, \mu_{\delta, G}$ with the rotation given by Eqs. (B.12) and (B.13). $\mu_{l *, G}$ and $\mu_{b, G}$ depend on the (unknown) distance. This is the reason that Table D. 1 gives the observed proper motions, not corrected for Galactic rotation and solar motion. $\mu_{\alpha *, v}$ and $\mu_{\delta, v}$ depend on the peculiar velocity $v$ of the pulsar and on the direction of this velocity.
Table 3. Values of constants defining coordinate transformations and velocity corrections.

\begin{tabular}{cccc}
\hline \hline \multicolumn{3}{c}{ Galactic pole, longitude node } \\
$\alpha_{\mathrm{GP}}=192.85948$ & $\delta_{\mathrm{GP}}=27.12825$ & $l_{\Omega}=32.93192$ & $\mathrm{a}$ \\
\hline \multicolumn{4}{c}{ Peculiar velocity Sun } \\
$U=10.0 \mathrm{~km} \mathrm{~s}^{-1} \quad V=5.3 \mathrm{~km} \mathrm{~s}^{-1}$ & $W=7.2 \mathrm{~km} \mathrm{~s}^{-1}$ & $\mathrm{~b}$ \\
\hline \multicolumn{4}{c}{ Galactic rotation } \\
$v_{R}\left(R_{0}\right)=v_{R}(R)=220 \mathrm{~km} \mathrm{~s}^{-1}$ & $\mathrm{~b}$ \\
\hline Distance Galactic center, scales pulsar distribution \\
$R_{0}=8.5 \mathrm{kpc}$ & $h=0.33 \mathrm{kpc}$ & $H=1.7 \mathrm{kpc}$ & $\mathrm{c}$ \\
\hline
\end{tabular}

Notes. For explanation of these constants see Appendices A and B. a: from Perryman et al. 1997, b: from Dehnen \& Binney 1998, c: from Verbiest et al. (2012).

\subsection{Best solution and fiducial intervals}

In the following sections we will discuss a number of models, and for each model compute a likelihood $L_{i}(\sigma)$ for an individual pulsar labelled $i$, as a function of the parameter vector $\sigma$. We then construct the deviance $\mathcal{L}$ with

$\mathcal{L}(\sigma)=-2 \sum_{i=1}^{N} \ln L_{i}(\sigma)$

where $N$ is the number of pulsars. $\sigma_{\text {opt }}$ is the parameter vector for which Eq. (11) reaches its minimum. We write differences with the optimal solution as

$\Delta \mathcal{L}(\sigma) \equiv \mathcal{L}(\sigma)-\mathcal{L}\left(\sigma_{\text {opt }}\right)$

For appropriate choices of $L_{i}$ these differences approximate a $\chi^{2}$ distribution. For a parameter vector consisting of a single parameter, we estimate its $68 \%$ range by determining for which values Eq. (12) is equal to 1 . To determine the range of values if the vector parameter has three parameters, we proceed as follows. We fix the value of one parameter at an offset from the optimal value, and then determine the combination of the two other parameters that gives the lowest value for $\Delta \mathcal{L}(\sigma)$. We vary the offset until this lowest value is 1 . Repeating this for each of the three parameters for positive and negative offsets from the best values gives the ranges listed in Table 4.

The best parameter values and the fiducial ranges determined this way do not depend on the normalization of $L_{i}$ : a constant multiplicative factor $x$ to any $L_{i}$ leads to a constant additive factor $-2 \ln x$ in Eq. (11) and drops out in Eq. (12). We will also use the deviance to compare different models, using

$\mathrm{d} \mathcal{L} \equiv \mathcal{L}^{a}\left(\sigma_{\text {opt }}^{a}\right)-\mathcal{L}^{b}\left(\sigma_{\text {opt }}^{b}\right)$,

where indices $a$ and $b$ refer to the different models. The distribution of $\mathrm{d} \mathcal{L}$ approximates a $\chi^{2}$ distribution less well than $\Delta \mathcal{L}$, but we will use this difference as a rough indication of relative merit of models.

\section{Maxwellian velocity distribution}

The Maxwellian velocity distribution is characterized by a single parameter $\sigma$ :

$f(v, \sigma) \mathrm{d} v=\sqrt{\frac{2}{\pi}} \frac{v^{2}}{\sigma^{3}} \exp \left[-\frac{v^{2}}{2 \sigma^{2}}\right] \mathrm{d} v ; \quad(0<v<\infty)$. 
F. Verbunt et al.: The observed velocity distribution of young pulsars

Table 4. Results of the model calculations for all 28 pulsars in our master list (A), and for the 19 youngest pulsars $\left(\tau_{\mathrm{c}}<10 \mathrm{Myr}\right.$, Y).

\begin{tabular}{|c|c|c|c|c|c|c|c|}
\hline & Sample & Single Maxwellian & & Two Maxwell & & & $v_{l}$ Gaussian \\
\hline & $N$ & $\begin{array}{ccc}\sigma & \text { range } & \mathrm{d} \mathcal{L} \\
\left(\mathrm{km} \mathrm{s}^{-1}\right) & \end{array}$ & $\begin{array}{c}\sigma_{1} \quad \text { range } \\
\left(\mathrm{km} \mathrm{s}^{-1}\right)\end{array}$ & $\begin{array}{cc}\sigma_{2} & \text { range } \\
\left(\mathrm{km} \mathrm{s}^{-1}\right)\end{array}$ & $\begin{array}{c}w \text { range } \\
(\%)\end{array}$ & & $\begin{array}{c}\sigma \quad \text { range } \\
\left(\mathrm{km} \mathrm{s}^{-1}\right)\end{array}$ \\
\hline Isot & 28 & $244 \quad 221-271 \quad \equiv 0$ & $77 \quad 62-97$ & $321 \quad 278-375$ & $42 \quad 29-54$ & -14 & $240 \quad 209-279$ \\
\hline Mixed models & A $22+6$ & $239 \quad 219-267 \quad-18$ & $75 \quad 61-95$ & $316 \quad 276-369$ & $42 \quad 30-55$ & -33 & \\
\hline c models & $\mathrm{Y} \quad 19$ & $277 \quad 247-314 \equiv 0$ & $83 \quad 62-117$ & $335 \quad 287-398$ & $32 \quad 17-47$ & -6 & $263 \quad 223-314$ \\
\hline Mixed models & Y $14+5$ & $273 \quad 245-310-16$ & $82 \quad 61-115$ & $328 \quad 285-391$ & $32 \quad 17-48$ & -22 & \\
\hline
\end{tabular}

Notes. For the mixed models we give separately the number of pulsars from a semi-isotropic and an isotropic distribution (see Table D.1). For each model we give the best parameters and their approximate $68 \%$ range determined by setting Eq. (12) to unity. Within each sample we also give the differences in deviance $\mathrm{d} \mathcal{L}$ (Eq. (13)) between each model and the model with a single isotropic Maxwellian, which gives an indication of their relative merits.

In the isotropic case, the Maxwellian may be decomposed in three independent Gaussians in any three mutually perpendicular directions. We choose the directions of increasing right ascension, increasing declination, and the radial direction. This enables us to write the joint probability of measured values $\varpi^{\prime}, \mu_{\alpha *}^{\prime}$, $\mu_{\delta}^{\prime}$ and actual values $D, v_{\alpha}=D \mu_{\alpha *, v}$ and $v_{\delta}=D \mu_{\delta, v}$ as

$$
\begin{aligned}
& P_{\operatorname{maxw}}\left(\varpi^{\prime}, \mu_{\alpha *}^{\prime}, \mu_{\delta}^{\prime}, D, v_{\alpha}, v_{\delta}, v_{r}\right)=G\left(v_{\alpha}, \sigma\right) G\left(v_{\delta}, \sigma\right) G\left(v_{r}, \sigma\right) \\
& \quad \times \frac{f_{D}(D)}{\int_{0}^{D_{\max }} f_{D}(D) \mathrm{d} D} \frac{1}{\sigma_{\varpi} \sqrt{2 \pi}} \exp \left[-\frac{\left(1 / D-\varpi^{\prime}\right)^{2}}{2 \sigma_{\varpi}^{2}}\right] \\
& \quad \times \frac{1}{\sigma_{\alpha} \sqrt{2 \pi}} \exp \left[-\frac{\left(\mu_{\alpha *, G}(D)+v_{\alpha} / D-\mu_{\alpha *}^{\prime}\right)^{2}}{2 \sigma_{\alpha}^{2}}\right] \\
& \quad \times \frac{1}{\sigma_{\delta} \sqrt{2 \pi}} \exp \left[-\frac{\left(\mu_{\delta, G}(D)+v_{\delta} / D-\mu_{\delta}^{\prime}\right)^{2}}{2 \sigma_{\delta}^{2}}\right]
\end{aligned}
$$

where

$$
G(v, \sigma)=\frac{1}{\sigma \sqrt{2 \pi}} \exp \left[-\frac{v^{2}}{2 \sigma^{2}}\right] ; \quad(-\infty<v<\infty) .
$$

To obtain the value of $\sigma$ which gives the most likely correspondence with the measurements, we must take into account contributions to the likelihood of all distances and velocities. We therefore define the likelihood for the Maxwellian as

$$
L_{\operatorname{maxw}}(\sigma)=\int_{0}^{D_{\max }} \int_{-\infty}^{\infty} \int_{-\infty}^{\infty} \int_{-\infty}^{\infty} P_{\operatorname{maxw}} \mathrm{d} v_{\alpha} \mathrm{d} v_{\delta} \mathrm{d} v_{r} \mathrm{~d} D .
$$

The radial velocities occur only in $G\left(v_{r}, \sigma\right)$, and thus the integral over $v_{r}$ can be computed separately: $\int_{-\infty}^{\infty} G\left(v_{r}, \sigma\right) \mathrm{d} v_{r}=1$. The integrals over $v_{\alpha}$ and $v_{\delta}$ are more involved, but can also be solved analytically. Thus, for $v_{\alpha}$

$$
\begin{aligned}
& \int_{-\infty}^{\infty} \exp \left\{-\frac{1}{2}\left[\frac{v_{\alpha}^{2}}{\sigma^{2}}+\frac{\left(v_{\alpha}+D\left(\mu_{\alpha *, G}-\mu_{\alpha *}^{\prime}\right)\right)^{2}}{D^{2} \sigma_{\alpha}^{2}}\right]\right\} \mathrm{d} v_{\alpha} \\
& =\sqrt{2 \pi}\left(\frac{1}{\sigma^{2}}+\frac{1}{D^{2} \sigma_{\alpha}^{2}}\right)^{-1 / 2} \exp \left[-\frac{1}{2} \frac{D^{2}\left(\mu_{\alpha *, G}-\mu_{\alpha *}^{\prime}\right)^{2}}{\sigma^{2}+D^{2} \sigma_{\alpha}^{2}}\right]
\end{aligned}
$$

and analogously for $v_{\delta}$. Taken together these results lead to

$$
L_{\operatorname{maxw}}(\sigma)=C \int_{0}^{D_{\max }} f_{D}(D) \exp \left[-\frac{\left(1 / D-\varpi^{\prime}\right)^{2}}{2 \sigma_{\varpi}^{2}}\right] \mathcal{I}_{\alpha} \mathcal{I}_{\delta} \mathrm{d} D
$$

where

$$
\begin{aligned}
C & \equiv\left[(2 \pi)^{3 / 2} \sigma_{\varpi} \sigma_{\alpha} \sigma_{\delta} \int_{0}^{D_{\max }} f_{D}(D) \mathrm{d} D\right]^{-1}, \\
\mathcal{I}_{\alpha} & \equiv\left(1+\frac{\sigma^{2}}{D^{2} \sigma_{\alpha}^{2}}\right)^{-1 / 2} \exp \left[-\frac{1}{2} \frac{\left(D \mu_{\alpha *, G}-D \mu_{\alpha *}^{\prime}\right)^{2}}{\sigma^{2}+D^{2} \sigma_{\alpha}^{2}}\right], \\
\mathcal{I}_{\delta} & \equiv\left(1+\frac{\sigma^{2}}{D^{2} \sigma_{\delta}^{2}}\right)^{-1 / 2} \exp \left[-\frac{1}{2} \frac{\left(D \mu_{\delta, G}-D \mu_{\delta}^{\prime}\right)^{2}}{\sigma^{2}+D^{2} \sigma_{\delta}^{2}}\right] .
\end{aligned}
$$

The integral over distances in Eq. (19) is computed numerically, out to $D_{\max }=10 \mathrm{kpc}$. $C$ ensures that each distribution in Eq. (15) is normalized to unity; in the computations $C$ may be ignored, as it only adds a constant in the deviance (Eq. (11)) and drops out in Eq. (12).

To illustrate the effect of the various factors in the integrand of Eq. (19) we show these separately in Fig. 2, for two pulsars. For a fixed velocity, the proper motion scales inversely with the distance. The large parallax of PSR 0034-0721, combined with its relatively small proper motion, favours a Maxwellian with a small average velocity, but still allows a Maxwellian with a high average velocity as this has a finite tail at low velocities. In contrast, the smaller parallax of PSR B1508+55 combined with its large proper motion, demands a Maxwellian with a large average velocity, because high velocities have vanishingly low probability in a Maxwellian with a low average velocity.

Labelling the likelihoods of Eq. (19) for each of $N$ pulsars with $i$, we compute the deviance $\mathcal{L}$ with Eq. (11). $\Delta \mathcal{L}(\sigma)$ (Eq. (12)) is shown for three pulsar samples in Fig. 3. The sample of all 28 pulsars in our master list (Table D.1) leads to $\sigma_{\text {opt }} \simeq 244 \mathrm{~km} \mathrm{~s}^{-1}$, with a range of about $50 \mathrm{~km} \mathrm{~s}^{-1}$ found from $\Delta \mathcal{L}=1$; see Table 4 . To illustrate the influence of a single pulsar, we also show $\Delta \mathcal{L}(\sigma)$ for the sample of 27 pulsars remaining after removing PSR B $1508+55$, the pulsar with the worst likelihood for $\sigma=245 \mathrm{~km} \mathrm{~s}^{-1}$. This sample has $\sigma_{\mathrm{opt}} \simeq 210 \mathrm{~km} \mathrm{~s}^{-1}$. The reason for this shift is evident from Fig. 2: the measurements of PSR B $1508+55$ require a large value of $\sigma$. Removing any one of the 27 other pulsars from the full sample leads to a much smaller shift.

The pulsar velocities of young pulsars, less affected by acceleration in the Galactic gravitational field, are more indicative of the pulsar velocities at birth, and therefore we also investigate the sample of the 19 youngest pulsars with characteristic age $\tau_{\mathrm{c}}<10 \mathrm{Myr}$. This leads to a higher optimal distribution parameter $\sigma_{\text {opt }} \simeq 280 \mathrm{~km} \mathrm{~s}^{-1}$. The smaller number of pulsars also leads to a wider range of $\sigma$ for which $\Delta \mathcal{L}(\sigma)<1$. An upper limit to $\tau_{\mathrm{c}}$ of $5 \mathrm{Myr}$ leads to the same $\sigma_{\mathrm{opt}}$ as for $10 \mathrm{Myr}$, but further widens the uncertainty range. Removing PSR B1508+55 

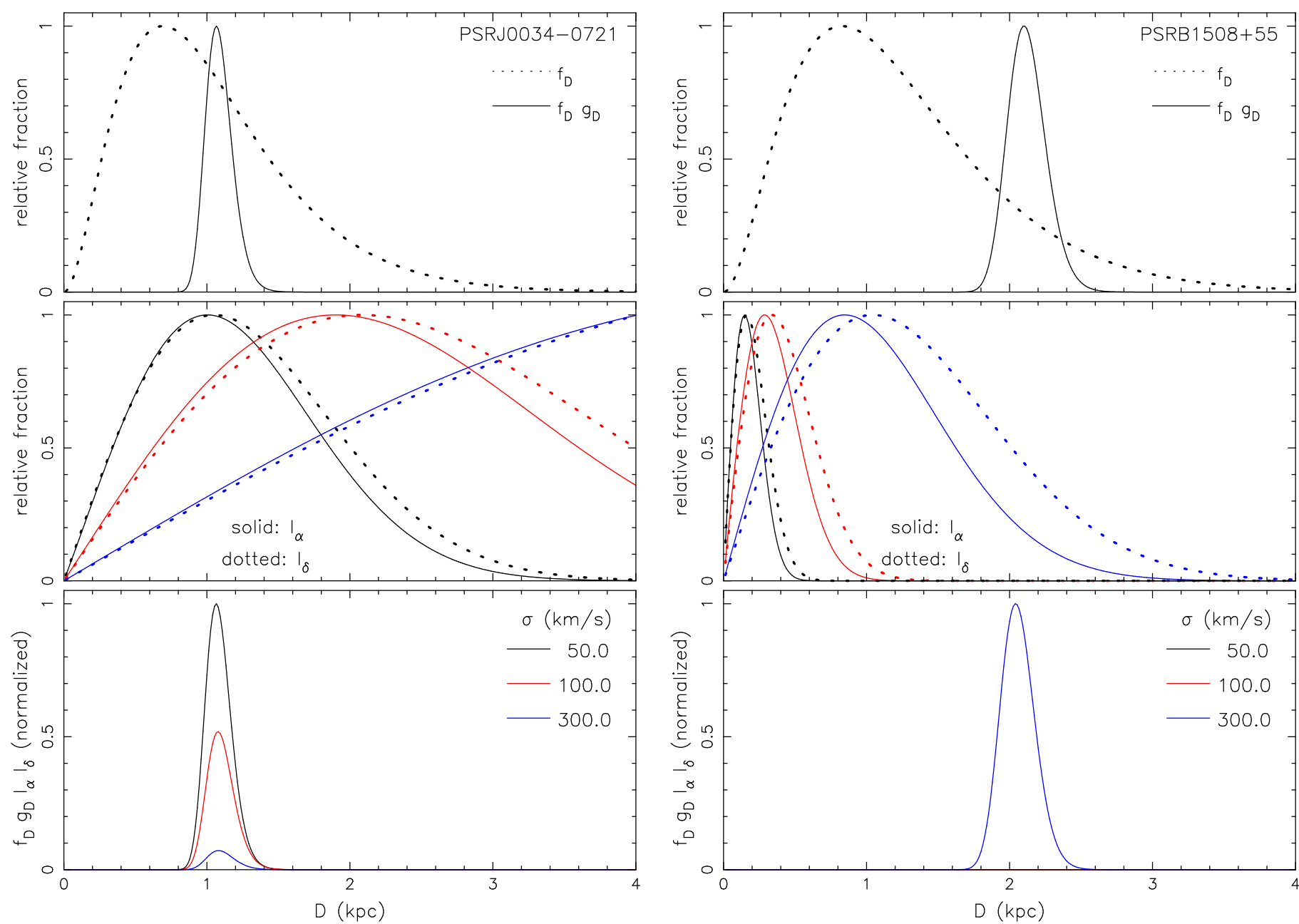

Fig. 2. Illustration for two pulsars of the contributions to the integrand of the likelihood $L_{\operatorname{maxw}}(\sigma)$ (Eq. (19)) of the separate factors $f_{D}(D) g_{D}\left(\varpi^{\prime} \mid D\right)$ (top graphs; Eqs. (1) and (4)), $I_{\alpha}$ and $I_{\delta}$ (middle graphs). Each curve has been normalized separately to maximum unity. $I_{\alpha}$ and $I_{\delta}$ are shown for for three values $\sigma$ of the Maxwellian (Eq. (14)). The lower graphs show the integrand of the likelihood $L_{\operatorname{maxw}}(\sigma)$ for three values of $\sigma$, as a function of distance, normalized to the highest maximum of the three. The measurements of PSR J0034-0721 (left) favour a low value of $\sigma$. Those of PSR B1508+55 (right) require a high value of $\sigma$, and the integrands for $\sigma=50$ and $100 \mathrm{~km} \mathrm{~s}^{-1}$ are indistinguishable from zero in this graph.

from the sample of young pulsars reduces the optimal distribution sample to $\sigma_{\mathrm{opt}} \simeq 235 \mathrm{~km} \mathrm{~s}^{-1}$.

Figures 1 and 2 indicate that a single Maxwellian is not a good description of the velocity distribution of young radio pulsars. We are therefore not unduly worried about the shifts in $\sigma_{\text {opt }}$ between the different samples, but move on to investigate more promising models.

\section{Sum of two Maxwellians}

We investigate a velocity distribution which is the sum of two Maxwellians, one to explain the lower observed velocities, and one for the higher velocities. Defining the vector of parameters $\boldsymbol{\sigma}=\left[\sigma_{1}, \sigma_{2}, w\right]$ we write

$f_{v}(v, \sigma) \mathrm{d} v=\sqrt{\frac{2}{\pi}} v^{2}\left[\frac{w}{\sigma_{1}^{3}} \exp \left(-\frac{1}{2} \frac{v^{2}}{\sigma_{1}^{2}}\right)+\frac{(1-w)}{\sigma_{2}^{3}} \exp \left(-\frac{1}{2} \frac{v^{2}}{\sigma_{2}^{2}}\right)\right] \mathrm{d} v$.

The likelihood for the sum of two Maxwellians is the sum of the likelihoods of the two Maxwellians: in analogy with Eq. (19) we have

$L_{2 \operatorname{maxw}}(\sigma)=w L_{\operatorname{maxw}}\left(\sigma_{1}\right)+(1-w) L_{\operatorname{maxw}}\left(\sigma_{2}\right)$.

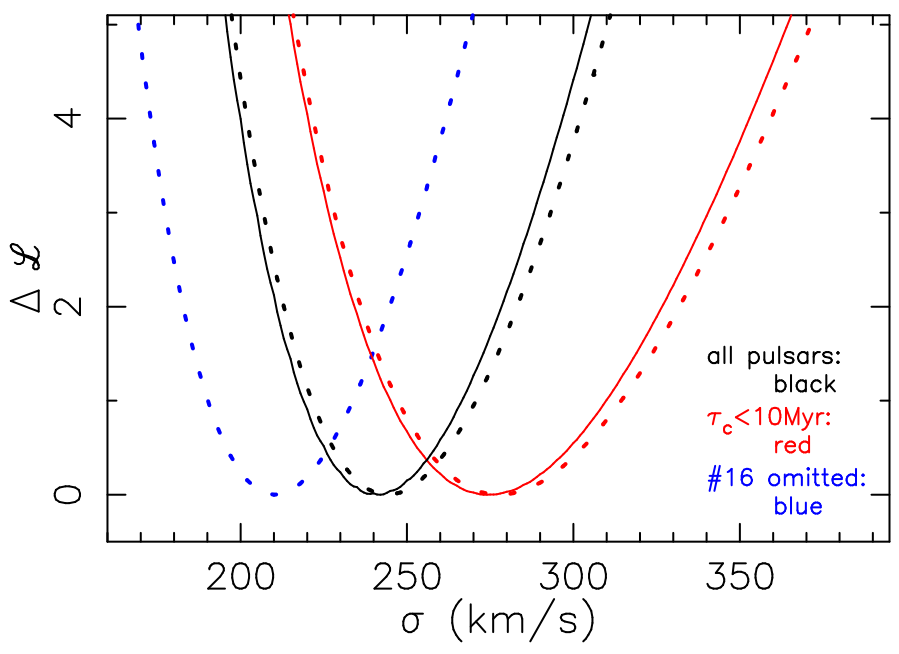

Fig. 3. Variation of $\mathcal{L}$ with velocity distribution parameter $\sigma$ for the model with a single isotropic Maxwellian (dotted lines), and for the mixed model in which most pulsars have an assumed semi-isotropic velocity distribution (solid lines). The colour coding indicates the pulsar sample: all 28 pulsars in our master list, the 27 pulsars remaining after removing PSR B1508+55, and the 19 youngest $(\tau<10 \mathrm{Myr}$ ) pulsars. 
F. Verbunt et al.: The observed velocity distribution of young pulsars

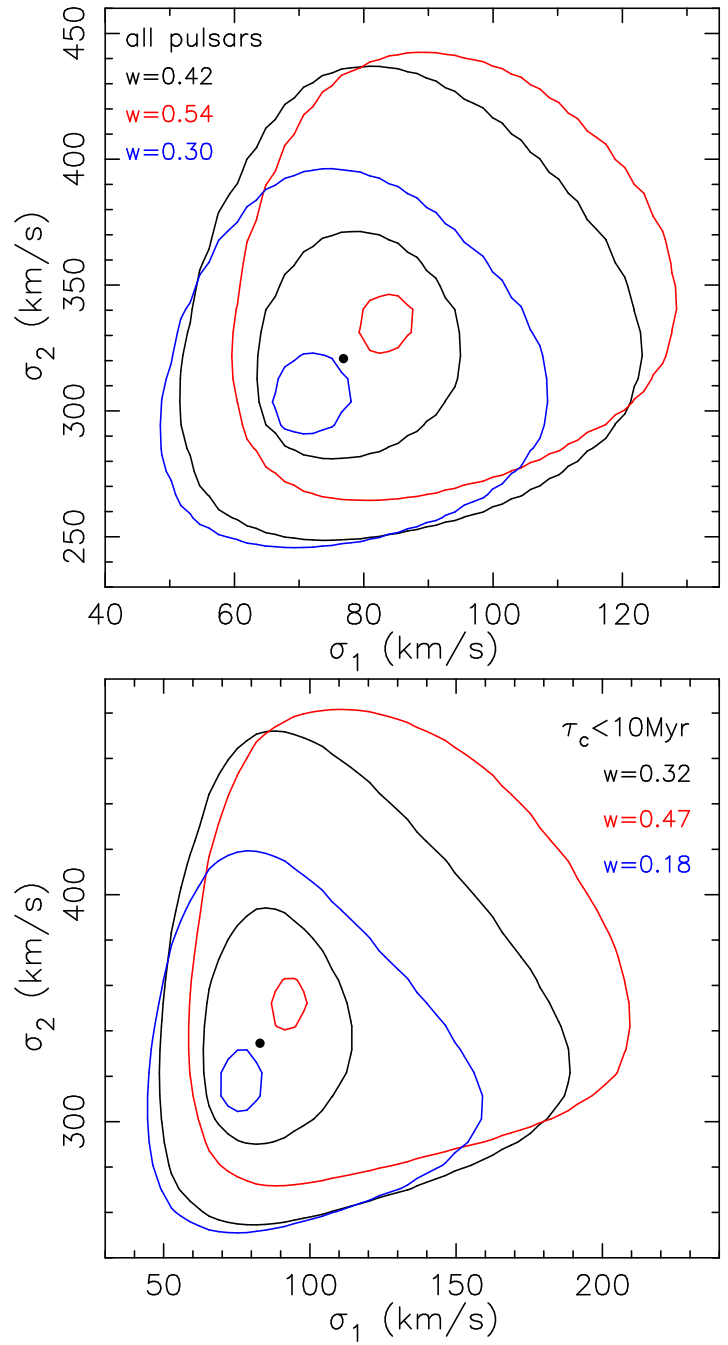

Fig. 4. Contours of $\mathcal{L}(\sigma)$ in three $\sigma_{1}, \sigma_{2}$ planes with fixed $w$, for the model with two isotropic Maxwellians. Contours of constant $\Delta \mathcal{L}(\sigma)$ (Eq. (12)) are shown for values 1 and 4, in each plane, The best solution is indicated with $\bullet$. Top: all pulsars. $\sigma_{\mathrm{opt}}=\left(77 \mathrm{~km} \mathrm{~s}^{-1}, 321 \mathrm{~km} \mathrm{~s}^{-1}\right.$, 0.42). Below: pulsars with $\tau_{\mathrm{c}}<10 \mathrm{Myr} . \sigma_{\mathrm{opt}}=\left(83 \mathrm{~km} \mathrm{~s}^{-1}, 335 \mathrm{~km} \mathrm{~s}^{-1}\right.$, $0.32)$.

We compute $L_{\operatorname{maxw}}(\sigma)$ on a grid of values of $\sigma$, in steps of $1 \mathrm{~km} \mathrm{~s}^{-1}$, and use the subroutine AMOEBA of Press et al. (1986), which implements the downhill simplex method of Nelder and Mead, to obtain the optimal values of $w, \sigma_{1}$ and $\sigma_{2}$ for which $\mathcal{L}$, computed from Eq. (21) with Eq. (11), has its minimum. The results are listed in Table 4, and illustrated in Fig. 4.

To decide on the significance of the second Maxwellian, we note that it adds two parameters to the model with one Maxwellian, and compute the deviance difference $\mathrm{d} \mathcal{L}$ with Eq. (13). We first investigate the sample of all 28 pulsars in our master list (sample A). For this sample, $\mathrm{d} \mathcal{L}=-14$, indicating that the addition of a second Maxwellian is very significant $\left(\Delta \chi^{2}=-14\right.$ corresponds to a $99.8 \%$ confidence level for two added parameters). The low-velocity component represents between $29 \%$ and $54 \%$ of the pulsar population. Figure 4 shows that the values of $\sigma_{1}$ and $\sigma_{2}$ are mildly correlated with $w$ : a larger (smaller) fraction of the low-velocity component leads to larger (smaller) values of $\sigma_{1}$ and $\sigma_{2}$. The shift, however, lies well within the error range of $\sigma_{1}$ and $\sigma_{2}$; the main effect of the correlation between $\sigma_{1}$ and $\sigma_{2}$ is to mitigate the drop of pulsar numbers with velocities between $\sigma_{1}$ and $\sigma_{2}$.

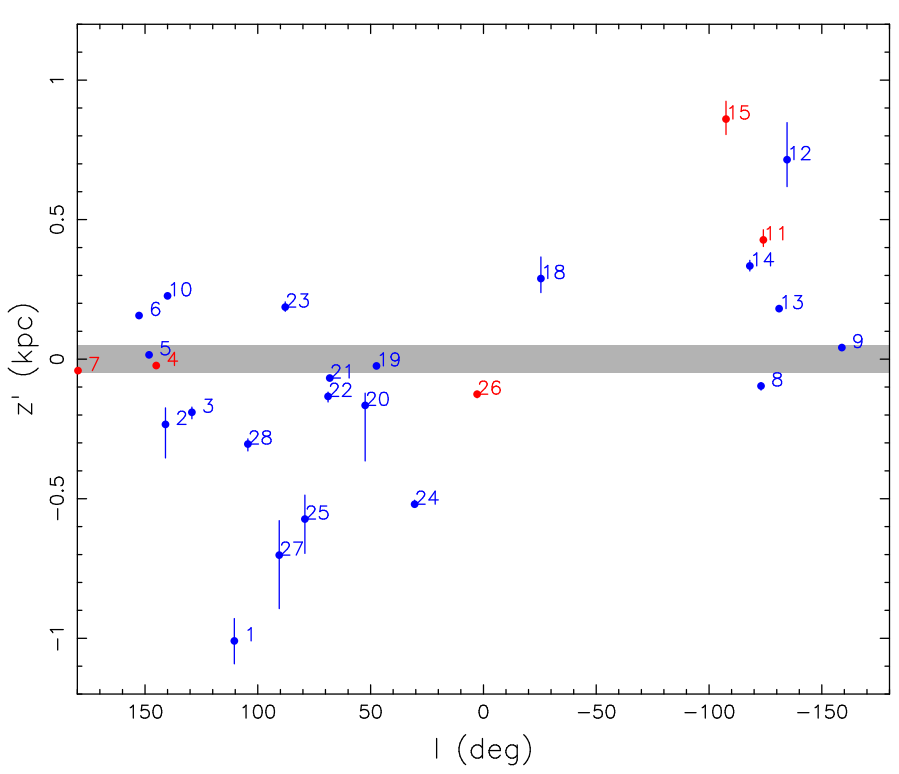

Fig. 5. Nominal distance from the Galactic plane $z^{\prime}=\sin b / \varpi^{\prime}$, range $\sin b /\left(\varpi^{\prime} \pm \sigma_{\varpi}\right)$, as a function of longitude. The blue points indicate pulsars nominally moving away from the plane, that is $z^{\prime}$ and $v_{z}^{\prime}=\mu_{b}^{\prime} \cos b / \varpi^{\prime}$ have the same sign; the red points are pulsars nominally moving towards the plane. The grey band indicates the scale height of $50 \mathrm{pc}$ of $\mathrm{O}$-stars. The numbers refer to the sequence number in Table D.1. Numbers 16 at $z^{\prime}=1.6 \mathrm{kpc}$ and 17 at $5.5 \mathrm{kpc}$, respectively, are outside the frame, and both are moving away from the Galactic plane.

The sample of 19 pulsars in our list with characteristic age $\tau_{\mathrm{c}}<10 \mathrm{Myr}$ (sample Y) leads to the same result, but with somewhat lager error margins for the parameters $\sigma$. For these young pulsars, the evidence for a second Maxwellian is still significant ( $\Delta \chi^{2}=-6$ is $95 \%$ confidence) .

\section{Semi-isotropic Maxwellian velocity distribution}

The isotropic Maxwellian velocity distribution has a major advantage in enabling us to compute three out of four integrals in Eq. (17) analytically. However, once the pulsar has moved away from the Galactic plane, we have more information, that we will put to use in this section: the pulsar velocity must be directed away from its place of birth, which for sufficiently large $|z|$ implies that $v_{z}>0$ when $z>0$ and $v_{z}<0$ when $z<0$. For these pulsars we assume an intrinsic distribution for the velocity which is an isotropic distribrution from which the velocities towards the Galactic plane have been removed: and refer to this distribution as semi-isotropic.

To quantify "sufficiently large" we show the nominal values of distance to the Galactic plane $z^{\prime}=D^{\prime} \sin b$ in Fig. 5, together with a band indicating the scale height of $\mathrm{O}$ stars, as a proxy for the place of birth of pulsars. Five pulsars in our list of 28 are moving towards the Galactic plane. Two of these, PSR B0329+54 (\#4 in our master list) and PSR J0538+2817 (\#7) are within the region where pulsars are born, and thus may well be moving towards the plane. PSR J2144-3933 (\#26) is the oldest pulsar in our sample, and may well be a returning pulsar. PSR B0818-13 (\#11) and PSR B1237+25 (\#15) are too young assuming their characteristic age is indicative of their real age to have reversed motion, and their motion towards the Galactic plane must be apparent. We may write $v_{z}$ as (see Fig. B.1)

$v_{z}=D \mu_{b} \cos b+v_{r} \sin b$. 
Hence a pulsar is moving away from the plane if

$z v_{z}>0$ if $v_{r}>\frac{-\mu_{b} \cos b}{\varpi \sin b}$.

Entering the nominal values $\varpi^{\prime}$ and $\mu_{b}^{\prime}$, we obtain $v_{r}>$ $120 \mathrm{~km} \mathrm{~s}^{-1}$ (\#11) and $v_{r}>12 \mathrm{~km} \mathrm{~s}^{-1}$ (\#15), indicating that these pulsars may well be moving as expected: away from the plane.

In computing for the case of semi-isotropic Maxwellians, we choose axes parallel to the (local) direction of right ascension and declination, and along the line of sight, and write the spatial velocity as

$\boldsymbol{v}=\left(v_{\alpha}, v_{\delta}, v_{r}\right)=\left(v \sin \xi_{1} \cos \xi_{2}, v \sin \xi_{1} \sin \xi_{2}, v \cos \xi_{1}\right)$

where

$0 \leq \xi_{1} \leq \pi ; \quad 0 \leq \xi_{2} \leq 2 \pi$

To determine which velocities lead to $v_{z}$ away from the Galactic plane, we first convert the velocities to Galactic coordinates using Eqs. (A.9), (A.10):

$\left(v_{l}, v_{b}, v_{r}\right)=\left(v \sin \xi_{1} \cos \left(\xi_{2}-\phi\right), v \sin \xi_{1} \sin \left(\xi_{2}-\phi\right), v \cos \xi_{1}\right)$,

where $\phi$ is given by Eq. (A.11). Entering $v_{b}$ and $v_{r}$ from Eq. (25) into (22) we obtain

$v_{z}=v\left[\sin \xi_{1} \sin \left(\xi_{2}-\phi\right) \cos b+\cos \xi_{1} \sin b\right]$.

Thus, the sign of $v_{z}$ does not depend on the speed $v$. The condition $v_{z}>0$ if $b>0$ and $v_{z}<0$ if $b<0$ may be written

$\sin \left(\xi_{2}-\phi\right)>\frac{-\tan b}{\tan \xi_{1}}$

We rewrite the joint probability of Eq. (15) for the semi-isotropic case as

$$
\begin{aligned}
& P_{\text {sim }}\left(\varpi^{\prime}, \mu_{\alpha *}^{\prime}, \mu_{\delta}^{\prime}, D, v, \xi_{1}, \xi_{2}\right)=0 \quad \text { if } \quad z v_{z}<0, \\
& P_{\text {sim }}\left(\varpi^{\prime}, \mu_{\alpha *}^{\prime}, \mu_{\delta}^{\prime}, D, v, \xi_{1}, \xi_{2}\right)=C \exp \left[-\frac{\left(1 / D-\varpi^{\prime}\right)^{2}}{2 \sigma_{\varpi}^{2}}\right] \\
& \quad \times \exp \left[-\frac{\left(\mu_{\alpha *, G}(D)+v \sin \xi_{1} \cos \xi_{2} / D-\mu_{\alpha *}^{\prime}\right)^{2}}{2 \sigma_{\alpha}^{2}}\right] \\
& \quad \times \exp \left[-\frac{\left(\mu_{\delta, G}(D)+v \sin \xi_{1} \sin \xi_{2} / D-\mu_{\delta}^{\prime}\right)^{2}}{2 \sigma_{\delta}^{2}}\right] \\
& \quad \times f_{D}(D) \sin \xi_{1} 2 \sqrt{\frac{2}{\pi}} \frac{v^{2}}{\sigma^{3}} \exp \left[-\frac{v^{2}}{2 \sigma^{2}}\right] \quad \text { if } \quad z v_{z}>0 .
\end{aligned}
$$

Here $C$ is defined with Eq. (19), and a factor 2 is added to normalize the semi-Maxwellian. The likelihood for the semiisotropic Maxwellian follows:

$L_{\text {sim }}(\sigma)=\int_{0}^{D_{\max }} \int_{0}^{2 \pi} \int_{0}^{\pi} \int_{0}^{\infty} P_{\text {sim }} \mathrm{d} v \mathrm{~d} \xi_{1} \mathrm{~d} \xi_{2} \mathrm{~d} D$.

Equation (26) shows that the condition that $v_{z}$ is in the correct direction is determined by the angles $\xi_{1}$ and $\xi_{2}$ and does not depend on $v$, and this allows the integral in Eq. (29) over the velocity to be done analytically. The integrals over the angles and distance are done numerically. Details are given in Appendix C.

\section{The mixed model}

In our mixed model we assume that the pulsars in the grey band in Fig. $5(\# 4,5,7,9,19)$ and the oldest pulsar (\#26) are drawn from an isotropic velocity distribution, whereas all others are drawn from a semi-isotropic distribution, in which the velocities towards the Galactic plane are excluded. The distribution parameter $\sigma$ for the semi-isotropic distribution is equal to the $\sigma$ for the isotropic distribution. In analogy with Eq. (11) we define the deviance for the mixed model as

$\mathcal{L}_{\text {mixed }}(\sigma)=-2\left(\sum_{i} \ln L_{\mathrm{sim}, i}(\sigma)+\sum_{j} \ln L_{\operatorname{maxw}, j}(\sigma)\right)$.

Where the sums over $i$ and over $j$ are for the pulsars whose velocity is drawn from a semi-isotropic distribution and an isotropic distribution, respectively. The best value for $\sigma$ is the value for which Eq. (30) reaches its minimum, and its range is determined from $\Delta \mathcal{L}=1$. The results are given in Table 4 and Fig. 3 , and are not very different from those for the single isotropic Maxwellian, both for sample A of all pulsars, and for sample $\mathrm{Y}$ for the youngest pulsars. For the $\mathrm{d} \mathcal{L}$ value it is seen that the mixed model is a significant improvement on the isotropic model. We return to this below, for the more interesting case of two Maxwellians.

In a more realistic model the semi-isotropic distribution is composed of two semi-Maxwellians, with the same distribution parameters $\sigma$ as the two isotropic Maxwellians that compose the isotropic distribution. In analogy with Eq. (21) we now have

$L_{2 \operatorname{sim}}(\sigma)=w L_{\text {sim }}\left(\sigma_{1}\right)+(1-w) L_{\text {sim }}\left(\sigma_{2}\right)$,

and in analogy with Eq. (30)

$\mathcal{L}_{2 \text { mixed }}(\boldsymbol{\sigma})=-2\left(\sum_{i} \ln L_{2 \operatorname{sim}, i}(\sigma)+\sum_{j} \ln L_{2 \operatorname{maxw}, j}(\sigma)\right)$,

where the sums over $i$ and over $j$ are for the pulsars whose velocity is drawn from a semi-isotropic distribution and an isotropic distribution, respectively. We use the subroutine AMOEBA of Press et al. (1986) to obtain the optimal values of $w, \sigma_{1}$ and $\sigma_{2}$ for which $\mathcal{L}_{2 \text { mixed }}$ has its minimum, and $\Delta \mathcal{L}=1$ for the range of these parameters. The results are given in Table 4 and Fig. 6.

The best values and the ranges for $\sigma_{1}, \sigma_{2}$ and $w$ for the semi-isotropic model are not significantly different from those of the isotropic model. Contour plots in the $\sigma_{1}-\sigma_{2}$ planes also are not significantly different from those for the model with two isotropic Maxwellians shown in Fig. 4.

The factor 2 in (the last line of) Eq. (28) ensures that the semi-Maxwellian is normalized to unity. As remarked above, a constant multiplicative factor for any likelihood drops out in Eq. (12), and thus does not affect the best solution and its range(s) within one model. However, to compare between models one must use the same normalizations of the separate distributions between the different models, and this requires the factor 2 in Eq. (28). The $\mathrm{d} \mathcal{L}$ values listed in Table 4 show that the mixed model is a highly significant improvement above the isotropic Maxwellian model, for the full sample A, and that it is still significant for sample $\mathrm{Y}$ of young pulsars.

It is interesting to look at this is some more detail. Suppose for the moment that the contributions to the integral of Eq. (17) are zero for $v_{z}$ velocities towards the plane, then the only difference between $L_{2 \operatorname{maxw}}(\sigma)$ and $L_{2 \operatorname{mixed}}(\sigma)$ is the multiplicative factor 2 in Eq. (28). In sample A for all pulsars, this affects only 
F. Verbunt et al.: The observed velocity distribution of young pulsars
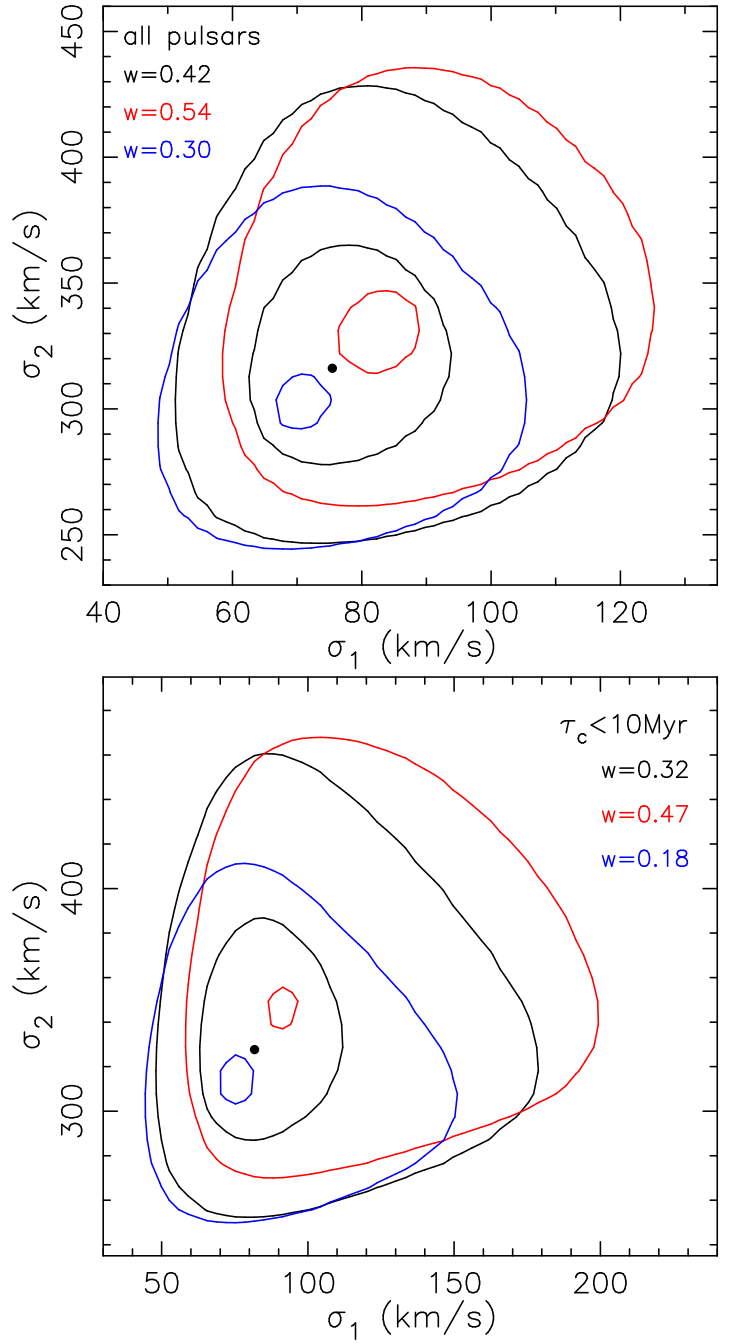

Fig. 6. As Fig. 4, now for the mixed model. $\sigma_{\mathrm{opt}}$ for all pulsars and for the youngest pulsars are listed in Table 4 .

the 22 pulsars for which a semi-Maxwellian applies, and leads to an added term in Eq. (32) equal to $-2 \times 22 \times \ln 2 \simeq-30.5$. In sample $\mathrm{Y} 14$ of the young pulsars are affected, leading to an added term $-2 \times 14 \times \ln 2 \simeq-19.4$. The actual differences $\mathrm{d} \mathcal{L}$ in deviance between the mixed models and purely isotropic models are smaller than this, which indicates that $v_{z}$ velocities towards the plane in fact do contribute to the integral of Eq. (17), also for pulsars for which such velocities are not expected. This implies that the isotropic model overestimates the likelihoods for these pulsars. PSR B0818-13 (\#11) is a case in point: its apparent $v_{z}^{\prime}$ velocity is towards the plane (Fig. 5), and thus $v_{z}$ velocities towards the plane may be expected to contribute noticeably to integral Eq. (17).

In Fig. 7 we show the ratio of the likelihoods for the mixed and isotropic two-Maxwellian model for each pulsar separately. The six pulsars whose velocities are drawn from an isotropic velocity distribution also in the mixed model by definition have a ratio of one of the likelihoods for the mixed and isotropic twoMaxwellian model. The eleven pulsars with ratios closest to the maximum possible, $1.8<L_{\text {mixed }} / L_{\text {iso }}<2$ say, are all young. For these pulsars, almost all velocities contributing to $L_{i}$ in the isotropic model contribute also in the mixed model. For 11 pulsars (sample A) or 3 pulsars (sample $\mathrm{Y}$ ) the velocity range that contributes to $L_{i}$ is restricted by the condition that $v_{z}$ be away

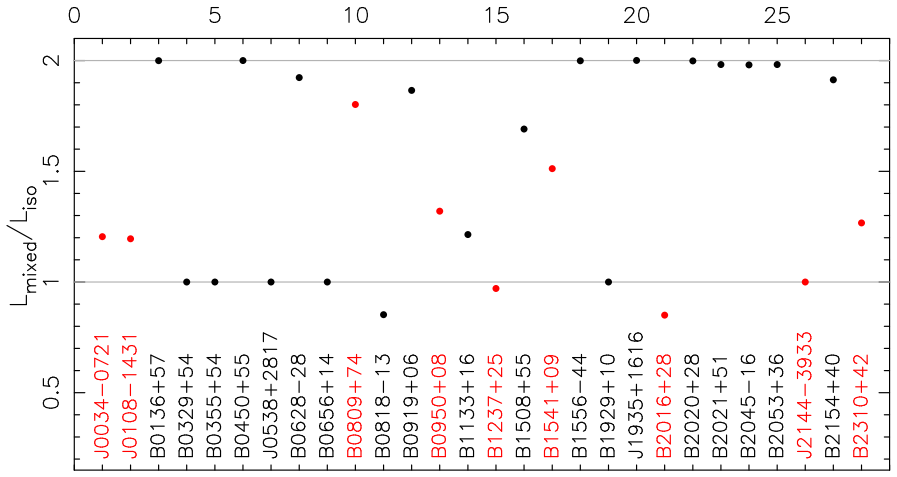

Fig. 7. Ratio of the likelihoods in the mixed and isotropic models shown for each pulsar. The red colour indicates old pulsars, with $\tau_{\mathrm{c}}>10 \mathrm{Myr}$. To illustrate the pure effect of the normalization of the velocity distribution, we use the same parameters $\sigma=\left(76 \mathrm{~km} \mathrm{~s}^{-1}, 318 \mathrm{~km} \mathrm{~s}^{-1}, 0.32\right)$ for both likelihoods. Use of $\sigma_{\text {opt }}$ for each model separately gives rise to small shifts.

from the Galactic plane, as shown by the difference of their $L_{\text {mixed }} / L_{\text {iso }}$ from the normalization factor 2 .

\section{The distribution of longitudinal velocities}

For comparison with earlier studies we also determine the model parameter $\sigma$ by only using the measurements of the parallax and the measurements $\mu_{1 *}^{\prime}$ of the proper motion in the direction of Galactic longitude. For this we choose the coordinates in the directions of Galactic longitude and latitude, and radial. We rewrite Eqs. (15) and (17) as

$$
\begin{aligned}
& P_{\text {gauss }}\left(\varpi^{\prime}, \mu_{l *}^{\prime}, D, v_{l}, v_{b}, v_{r}\right)=C_{l} f_{D}(D) G\left(v_{l}, \sigma\right) G\left(v_{b}, \sigma\right) G\left(v_{r}, \sigma\right) \\
& \quad \times \exp \left[-\frac{\left(1 / D-\varpi^{\prime}\right)^{2}}{2 \sigma_{\varpi}^{2}}\right] \\
& \quad \times \exp \left[-\frac{\left(\mu_{l *, G}(D)+v_{l} / D-\mu_{l *}^{\prime}\right)^{2}}{2 \sigma_{l}^{2}}\right]
\end{aligned}
$$

where

$C_{l} \equiv\left[2 \pi \sigma_{\varpi} \sigma_{l} \int_{0}^{D_{\max }} f_{D}(D) \mathrm{d} D\right]^{-1}$,

and

$L_{\text {gauss }}(\sigma)=\int_{0}^{D_{\max }} \int_{-\infty}^{\infty} \int_{-\infty}^{\infty} \int_{-\infty}^{\infty} P_{\text {gauss }} \mathrm{d} v_{l} \mathrm{~d} v_{b} \mathrm{~d} v_{r} \mathrm{~d} D$.

$\mu_{l *}$ and its error $\sigma_{\mu}$ are obtained from $\mu_{\alpha^{*}}, \mu_{\delta}$ and their errors with Eq. (A.9). The integrals over $v_{b}$ and $v_{r}$ are decoupled from the other integrals, and equal to 1. Equation (34) is rewritten:

$L_{\text {gauss }}(\sigma)=C_{l} \int_{0}^{D_{\max }} f_{D}(D) \exp \left[-\frac{\left(1 / D-\varpi^{\prime}\right)^{2}}{2 \sigma_{\varpi}{ }^{2}}\right] \mathcal{I}_{l} \mathrm{~d} D$,

where

$\mathcal{I}_{l} \equiv\left(1+\frac{\sigma^{2}}{D^{2} \sigma_{l}^{2}}\right)^{-1 / 2} \exp \left[-\frac{1}{2} \frac{\left(D \mu_{l *, G}-D \mu_{l *}^{\prime}\right)^{2}}{\sigma^{2}+D^{2} \sigma_{l}^{2}}\right]$.

In this case, there is no difference between the isotropic and mixed model, because $v_{z}$ does not affect $v_{l}$. We compute the deviance (Eq. (11)) with Eq. (35), to determine the values $\sigma_{\mathrm{opt}}$ for which the deviance reaches its minimum, and their range 
Table 5. Comparison of the results of our best model with those obtained in some earlier studies.

\begin{tabular}{l|cc|cccccc}
\hline \hline & \multicolumn{2}{|c|}{ Single Maxwellian } & \multicolumn{5}{c}{ Two Maxwellians } \\
\hline Reference & $\sigma$ & $\begin{array}{c}\text { range } \\
\left(\mathrm{km} \mathrm{s}^{-1}\right)\end{array}$ & $\begin{array}{c}\sigma_{1} \\
\left(\mathrm{~km} \mathrm{~s}^{-1}\right)\end{array}$ & $\begin{array}{c}\text { range } \\
\left(_{2}\right.\end{array}$ & $\begin{array}{c}\text { range } \\
\left(\mathrm{km} \mathrm{s}^{-1}\right)\end{array}$ & $\begin{array}{c}w \\
\text { range } \\
(\%)\end{array}$ \\
\hline $\begin{array}{l}\text { Arzoumanian et al. (2002) } \\
\text { Brisken et al. (2003a) }\end{array}$ & 290 & $260-320$ & 90 & $75-110$ & 500 & $350-750$ & 40 & $20-60$ \\
Hobbs et al. (2005) & 265 & $239-291$ & 99 & & 294 & & 20 & \\
Faucher-Giguère \& Kaspi (2006) & 290 & $260-320$ & 160 & $130-180$ & 780 & $640-930$ & 90 & $87-100$ \\
Mixed model sample A & 239 & $219-267$ & 75 & $60-95$ & 316 & $276-368$ & 42 & $30-52$ \\
\hline
\end{tabular}

Notes. ${ }^{(a)}$ Arzoumanian et al. (2002) fit Gaussians; comparison of their Eq. (1) with our Eq. (20) shows that these are components of Maxwellians. Thus, their $\sigma$ values may be compared directly with those in the other papers, contrary to the statement by Brisken et al. (2003a, below their Eq. (3)).

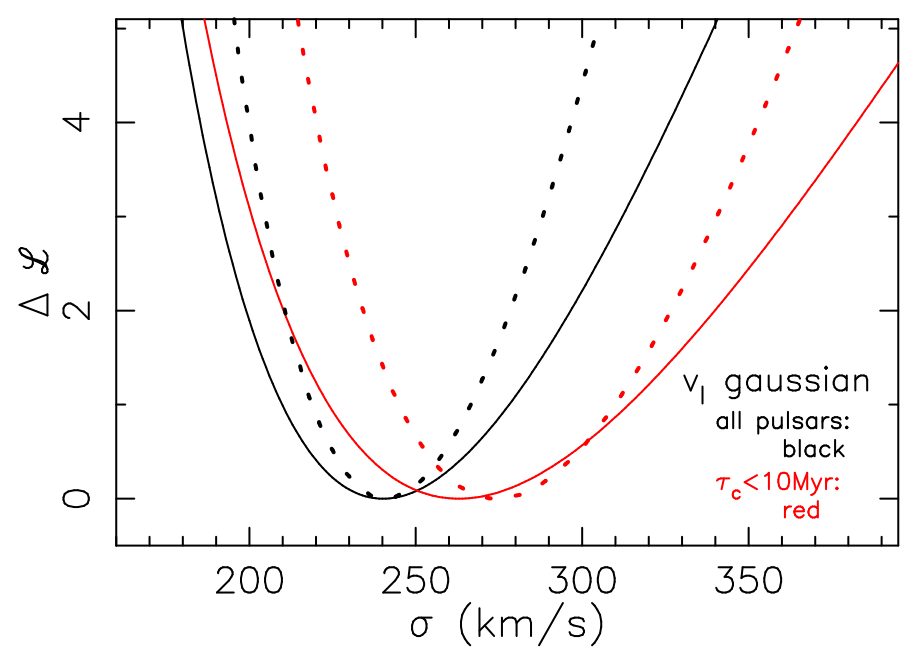

Fig. 8. Variation of $\mathcal{L}$ with $\sigma$ when only measurements of the parallax $\varpi^{\prime}$ and of the proper motion $\mu_{l *}^{\prime}$ in the direction of Galactic longitude are used (solid lines). For comparison the results for the mixed model, that uses parallaxes and both proper motions $\mu_{\alpha *}, \mu_{\delta}$ are also shown (dotted lines).

from $\Delta \mathcal{L}=1$. The results are listed in Table 4 and shown in Fig. 8. Interestingly, PSR B1508+55 is not an outlier in $v_{l}$ : its proper motion is almost completely in the direction of Galactic latitude (see Fig. 1). For sample A (all pulsars), $\sigma_{\text {opt }}$ is the same as for the isotropic or semi-isotropic single Maxwellian; for sample $\mathrm{Y}$ (youngest pulsars) it is marginally lower. The limitation to only one component of the proper motion leads to a reduced accuracy of $\sigma_{\mathrm{opt}}$, as expected. As a consequence the superposition of two Gaussians (that is components of two Maxwellians in the direction of Galactic longitude) does not improve significantly over the single Maxwellian description $\left(\sigma=109 \mathrm{~km} \mathrm{~s}^{-1}, 277 \mathrm{~km} \mathrm{~s}^{-1}, 0.27, \mathrm{~d} \mathcal{L}=1\right)$.

\section{Conclusions and discussion}

Previous work derived the velocity distribution of pulsars from the observed distances and proper motions, and then compared this distribution with model distributions. This reduces the information present in the observations, complicates error propagation, and has lead to wrong likelihood definitions. The uncertainties in the proper motions determined from timing are two to three orders of magnitude larger than those of the proper motions in our master list, that are determined from VLBI. The larger number of such proper motions (less than one order of magnitude) does not make up for their larger uncertainties, so that inclusion of these proper motions does not significantly improve the analysis. The use of distances determined from dispersion measures further complicates the analysis, because the related distance uncertainties are dominated by systematic effects, and cannot be described with a Gaussian, even in approximation.

Our approach is more reliable because we a) derive predictions for the observed parameters (parallax and proper motion) from the model, and compare these directly with the relevant measurements; b) only use VLBI determinations from after 2000 of both parallax and proper motion, whose uncertainties are well described with Gaussians; and c) include the intrinsic Galactic distribution of pulsars (as expressed in $f_{D}(D)$, Eq. (4)). Our mixed model furthermore takes into account that velocity component $v_{z}$ perpendicular to the Galactic plane of a young pulsar well away from that plane must be in the direction away from the plane.

Applying this to the pulsars in our master list, we find that the description of the velocity distribution of the pulsars with two Maxwellians improves significantly on the description with a single Maxwellian. Our model describing $v_{l}$ with a single Gaussian gives a similar value for $\sigma$ as the (mixed or isotropic) single Maxwellian, as expected for an isotropic velocity distribution. Comparison with earlier results, compiled in Table 5, shows that our more accurate method leads to more accurately determined model parameters. We show in Fig. 1 that our best solution corresponds well with the observed distribution of $v_{\perp}$. One would be tempted to conclude that our whole analysis apparatus can be replaced with a straightforward fit of the cumulative $v_{\perp}$ according to the model to the observed cumulative data for $v_{\perp}^{\prime}$ ! The reasons for the succes of the simpler method are the relatively small errors in the parallax, which limit the importance of $f_{D}(D)$, and the smallness of the correction for Galactic rotation with respect to the observed proper motions: $\mu_{\alpha *, G} \ll \mu_{\alpha *}^{\prime}$ and $\mu_{\delta, G} \ll \mu_{\delta}^{\prime}$ (Fig. 1). Indeed, ignoring the corrections for Galactic rotation hardly affects the results (Verbunt \& Cator 2017). Corrections for Galactic motion matter only for distances much larger than those of the pulsars in our master list.

Whereas our results indicate that the available velocity measurements are adequately described by the sum of two (isotropic or semi-isotropic) Maxwellians, they do not prove that the actual distribution is given by this model, in preference to for example (sums of) isotropic Gaussians, Paczyński functions (see Eqs. (4), (5) of Paczyński 1990), or exponentials - all of which incidentally peak at zero velocity. Indeed, Maxwellians may be somewhat unlikely, as the condition required for the Maxwellian distribution in a mono-atomic gas, that is continuous redistribution of kinetic energy by frequent elastic collisions between atoms, 
certainly is not met by young pulsars. Our results do indicate that the velocity distribution is wide, with relative contributions of higher and lower velocities which appear incompatible with a single Gaussian, Paczyński function or exponential. The adequateness of two Maxwellians for the description of the currently available data (as suggested by Fig. 1) implies that more velocity data are necessary to discriminate between this model and models that use other functional forms.

With the exception of Brisken et al. (2003a), who do not give error estimates, all previous authors find significantly higher velocities for the high-velocity component than we do. The compilation in Table 5 illustrates that the fraction of pulsars in the high-velocity component $(1-w)$ is inversely related to the characteristic velocity of that component. A small number of erroneously very high velocities leads to a high value of $\sigma_{2}$. Because the combination of $\sigma_{2}>500 \mathrm{~km} \mathrm{~s}^{-1}$ with a low value of $w$, thus high $1-w$, would lead to a much higher fraction of pulsars with $v_{\perp}>370 \mathrm{~km} \mathrm{~s}^{-1}$, say, than observed, the high value of $\sigma_{2}$ forces a high value of $w$. We suggest that the higher velocities derived by previous authors are affected by the inclusion of unreliable distances determined from dispersion measures. In the case of Arzoumanian et al. (2002) we note that all parallaxes are from before 2000, that is not corrected for differential ionospheric refraction. As Hartman (1997) has shown, underestimating velocity errors leads to overestimating velocities.

The analysis by Hobbs et al. (2005) is based on the nominal velocities $v_{\perp}^{\prime}=\mu_{\perp}^{\prime} / \varpi^{\prime}$, and does not take into account the large errors in both distances and proper motions of their sample. These errors blur the intrinsic distribution. We suggest that this prevents Hobbs et al. from recognising the presence of low velocities, and from recovering a bimodal velocity distribution in their analysis. The best model with two velocity components by Faucher-Giguère \& Kaspi (2006) allows $w=1$, that is the second component is not significant. Our analysis in Sect. 8 suggests that this is due to their small sample size (34 pulsars, of which only eight have a measured parallax).

Our results imply that the velocity contrast between the lowand high-velocity components is a factor 3 to 6 , and that 30 to $50 \%$ of the pulsars arise from the low-velocity component. It has been suggested (Podsiadlowksi et al. 2004) that pulsars formed from small iron cores or via electron capture would have a lower kick velocity than those formed from higher-mass core collapse, which may lead to a bimodal velocity distribution of pulsars. The existence of a class of neutron stars with low birth velocity has been derived from the properties of Be X-ray binaries (Pfahl et al. 2002) and the properties of millisecond pulsars binaries (Van den Heuvel 2004).

The fact that some pulsars are born in binaries and others from single stars will also affect the velocity distribution of single pulsars. Whether the observed bimodal velocity distribution reflects these different origins can be investigated in a population synthesis.

One of the goals of our work was to determine the fraction of pulsars with velocities small enough to remain bound to a globular cluster, or in a binary. In Fig. 9 we show the fraction of pulsars with velocity less than $v$ as a function of $v$. For a typical escape velocity of a globular cluster, $60 \mathrm{~km} \mathrm{~s}^{-1}$ say, it is seen that this fraction is about $5 \%$ in our best model (mixed, for sample A). It varies from about $3 \%$ to about $7.5 \%$ in the range of $\sigma_{1}$. At these low velocities, the fraction of pulsars is dominated completely by the low-velocity component, and therefore varies linearly with $w$ for fixed $\sigma_{1}$ and $\sigma_{2}$.

Finally, we mention two reasons why the determination of pulsar velocities from a local sample may lead to an
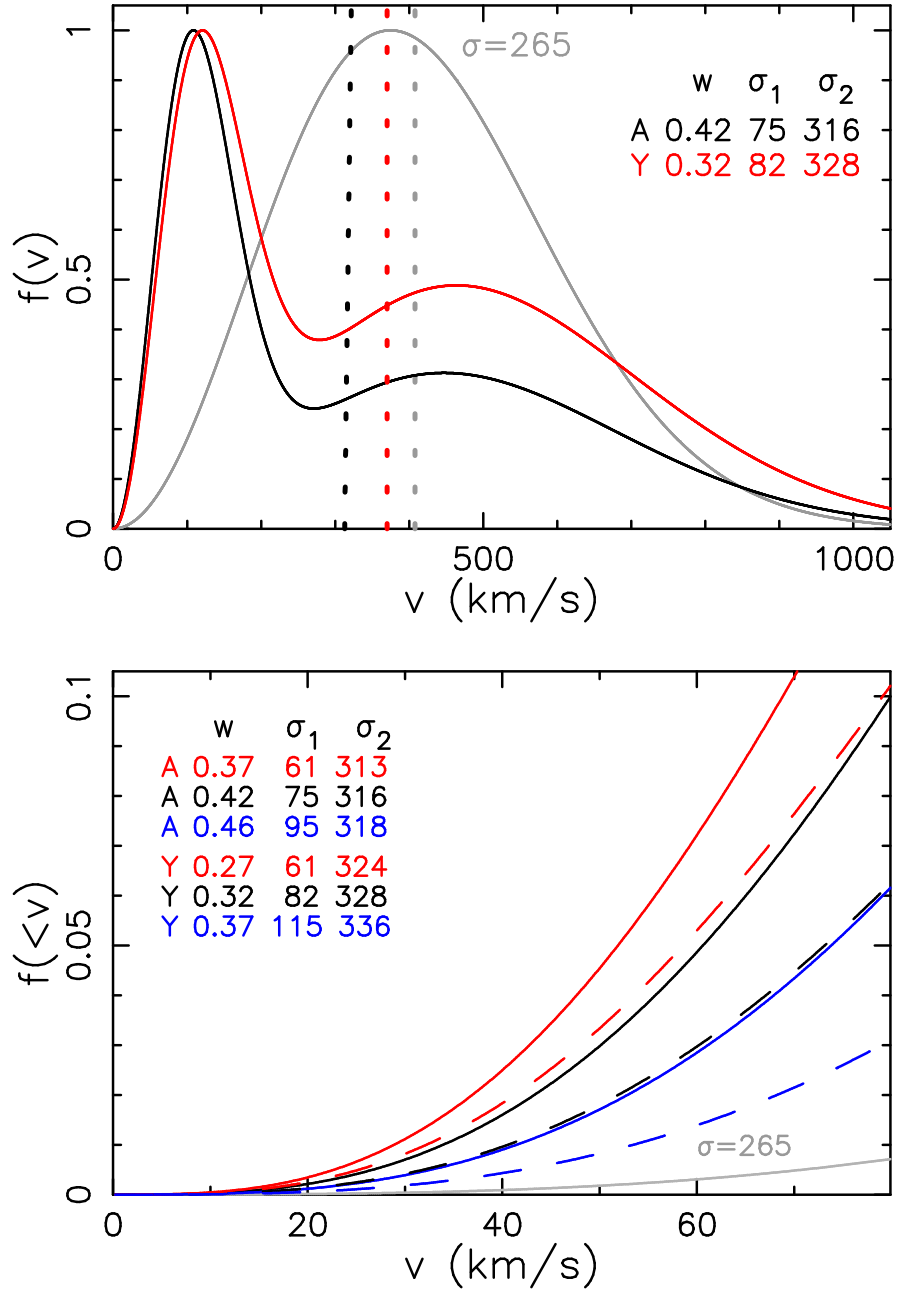

Fig. 9. Top: our best velocity distribution for all pulsars and for the youngest pulsars, together with a single Maxwellian. The vertical dotted lines indicates the median velocities: 313,370 and $408 \mathrm{~km} \mathrm{~s}^{-1}$. Below: fraction $f(<v)$ of pulsars with velocity less than $v$, for the best mixed model for all pulsars (black), and for the lowest and highest value in the range of $\sigma_{1}$ (red, blue). Solid lines: all pulsars; dashed lines: pulsars with $\tau_{\mathrm{c}}<10 \mathrm{Myr}$. In grey we show the fraction for a single Maxwellian (Hobbs et al. 2005).

underestimate of the average velocity. The first one is Galactic drift: motion in the Galactic gravitational potential leads to reduction of the velocity of a pulsar that moves away from the center of the galaxy, and an increase if it moves towards the center. Thus if pulsars with an origin closer to the Galactic center contribute more to the locally observed sample than pulsars with an origin further out, the locally measured velocity distribution underestimates the distribution at birth (Hansen \& Phinney 1997). The second reason is related to the velocity perpendicular to the plane: pulsars with a high $\left|v_{z}\right|$ move further from the plane, and thus must have a higher luminosity to be detected. In a flux-limited sample this leads to an over-representation of the low-velocity pulsars. These effects can be studied best in a population synthesis that takes these and other selection effects into account. Since such a synthesis involves also a larger number of parameters, a first step would be the measurement of more pulsar distances and proper motions.

Acknowledgements. We thank Gijs Nelemans for useful discussions. 


\section{References}

Arzoumanian, Z., Chernoff, D. F., \& Cordes, J. M. 2002, ApJ, 568, 289 Bailer-Jones, C. A. L. 2015, PASP, 127, 994

Brisken, W. F., Benson, J. M., Beasley, A. J., et al. 2000, ApJ, 541, 959

Brisken, W. F., Benson, J. M., Goss, W. M., \& Thorsett, S. E. 2002, ApJ, 571, 906

Brisken, W. F., Fruchter, A. S., Goss, W. M., Herrnstein, R. M., \& Thorsett, S. E. 2003a, AJ, 126, 3090

Brisken, W. F., Thorsett, S. E., Golden, A., \& Goss, W. M. 2003b, ApJ, 593, L89

Chatterjee, S., Cordes, J. M., Lazio, T. J. W., et al. 2001, ApJ, 550, 287

Chatterjee, S., Cordes, J. M., Vlemmings, W. H. T., et al. 2004, ApJ, 604, 339

Chatterjee, S., Brisken, W. F., Vlemmings, W. H. T., et al. 2009, ApJ, 698, 250

Dehnen, W., \& Binney, J. J. 1998, MNRAS, 298, 387

Deller, A. T., Tingay, S. J., Bailes, M., \& Reynolds, J. E. 2009, ApJ, 701, 1243

Faucher-Giguère, C.-A., \& Kaspi, V. M. 2006, ApJ, 643, 332

Hansen, B. M. S., \& Phinney, E. S. 1997, MNRAS, 291, 569

Hartman, J. W. 1997, A\&A, 322, 127

Hobbs, G., Lyne, A. G., Kramer, M., Martin, C. E., \& Jordan, C. 2004, MNRAS, 353,1311

Hobbs, G., Lorimer, D. R., Lyne, A. G., \& Kramer, M. 2005, MNRAS, 360, 974 Igoshev, A., Verbunt, F., \& Cator, E. 2016, A\&A, 591, A123
Kirsten, F., Vlemmings, W., Campbell, R. M., Kramer, M., \& Chatterjee, S. 2015, A\&A, 577, A111

Lane, A. P. 1979, PASP, 91, 405

Lutz, T. E., \& Kelker, D. H. 1973, PASP, 85, 573

Manchester, R. N., Hobbs, G. B., Teoh, A., \& Hobbs, M. 2005, AJ, 129, 1993

Paczyński, B. 1990, ApJ, 348, 485

Perryman, M. A. C., \& ESA 1997, The HIPPARCOS and TYCHO catalogues. Astrometric and photometric star catalogues derived from the ESA HIPPARCOS Space Astrometry Mission, ESA SP, 1200

Pfahl, E., Rappaport, S., Podsiadlowski, P., \& Spruit, H. 2002, ApJ, 574, 364

Podsiadlowski, P., Langer, N., Poelarends, A. J. T., et al. 2004, ApJ, 612, 1044

Press, W. H., Flannery, B. P., Teukolsky, S. A., \& Vetterling, W. T. 1986 ,

Numerical recipes: the art of scientific computing (Cambridge University Press)

Smart, W. M. 1938, Stellar dynamics (Cambridge University Press)

van den Heuvel, E. P. J. 2004, in 5th INTEGRAL Workshop on the INTEGRAL Universe, eds. V. Schoenfelder, G. Lichti, \& C. Winkler, ESA SP, 552, 185

Verbiest, J. P. W., Weisberg, J. M., Chael, A. A., Lee, K. J., \& Lorimer, D. R. 2012, ApJ, 755, 39

Verbunt, F., \& Cator, E. 2017, J. Astrophys. Astron., 38, 40

Yao, J. M., Manchester, R. N., \& Wang, N. 2017, ApJ, 835, 29 


\section{Appendix A: Transformations of equatorial to Galactic coordinates}

For the convenience of the reader we summarize the equations for coordinate transformations that we use. Lane (1979) gives (two of the three) equations for conversion from Galactic to equatorial for B1950.0. He notes that the equatorial coordinates of the Galactic pole $\alpha_{\mathrm{GP}}, \delta_{\mathrm{GP}}$ and the Galactic longitude $l_{\Omega}$ of the node where the Galactic plane $(b=0)$ crosses the equator, define the coordinate transformation and thus also the equatorial coordinates of the centre $l=b=0$. We note that this centre does not coincide exactly with the actual centre of the galaxy (for example as defined by $\mathrm{Sgr} \mathrm{A}^{*}$ ). We give all three equations, rewriting them slightly to show explicitly the role of $\alpha_{\mathrm{GP}}, \delta_{\mathrm{GP}}$, and $l_{\Omega}$. The coordinate transformation is composed of three rotations: one around the Galactic $z$-axis to bring the Galactic centre to the node (this replaces $l$ with $l-l_{\Omega}$ ), one around the equatorial $z$-axis to bring the spring node to the node (this replaces $\alpha$ with $\alpha-\alpha_{\Omega}=\alpha-\left(\alpha_{\mathrm{GP}}+\frac{\pi}{2}\right)$, and finally around the now common $x$-axis over an angle $\frac{\pi}{2}-\delta_{\mathrm{GP}}$ to align the Galactic pole with the equatorial pole. The resulting equations are (see also Lane 1979),

$$
\begin{aligned}
\cos \left(\alpha-\alpha_{\mathrm{GP}}-\frac{\pi}{2}\right) \cos \delta= & \cos \left(l-l_{\Omega}\right) \cos b \\
\sin \left(\alpha-\alpha_{\mathrm{GP}}-\frac{\pi}{2}\right) \cos \delta= & \cos \left(\frac{\pi}{2}-\delta_{\mathrm{GP}}\right) \sin \left(l-l_{\Omega}\right) \cos b \\
& -\sin \left(\frac{\pi}{2}-\delta_{\mathrm{GP}}\right) \sin b, \\
\sin \delta= & \sin \left(\frac{\pi}{2}-\delta_{\mathrm{GP}}\right) \sin \left(l-l_{\Omega}\right) \cos b \\
& +\cos \left(\frac{\pi}{2}-\delta_{\mathrm{GP}}\right) \sin b .
\end{aligned}
$$

To find the equatorial coordinates $\alpha_{\mathrm{GC}}, \delta_{\mathrm{GC}}$ for the centre of the coordinate system, we enter $l=b=0$ and combine Eqs. (A.1), (A.2) to find:

$\tan \left(\alpha_{\mathrm{GC}}-\alpha_{\mathrm{GP}}-\frac{\pi}{2}\right)=\frac{\cos \left(\frac{\pi}{2}-\delta_{\mathrm{GP}}\right) \sin \left(-l_{\Omega}\right)}{\cos \left(-l_{\Omega}\right)}$,

$\sin \delta_{\mathrm{GC}}=\sin \left(\frac{\pi}{2}-\delta_{\mathrm{GP}}\right) \sin \left(-l_{\Omega}\right)$.

Perryman et al. (1997) give the pole and node longitude for J2000.0 as

$\alpha_{\mathrm{GP}}=192: 85948, \quad \delta_{\mathrm{GP}}=27.12825, \quad l_{\Omega}=32 \circ 93192$.

and with Eqs. (A.1)-(A.3), these define the coordinate transformation for J2000.0 in the ICRS system. Entering these values in Eqs. (A.4), (A.5) we find

$\alpha_{\mathrm{GC}}=266.40500, \quad \delta_{\mathrm{GC}}=-28.93617$.

For later reference we combine Eqs. (A.1), (A.2) for the Galactic center $l=b=0$ into

$\tan \left(-l_{\Omega}\right)=\frac{\sin \left(\alpha_{\mathrm{GC}}-\alpha_{\mathrm{GP}}-\frac{\pi}{2}\right) / \cos \left(\frac{\pi}{2}-\delta_{\mathrm{GP}}\right)}{\cos \left(\alpha_{\mathrm{GC}}-\alpha_{\mathrm{GP}}-\frac{\pi}{2}\right)}$,

and verify that entering the coordinates for pole and centre from Eqs. (A.6), (A.7) in Eq. (A.8) we re-obtain $l_{\Omega}$ correctly.

The next step is to determine the transformation of the proper motions. This is done by Smart (1938, Chap. 1.41), who notes that it corresponds to a rotation over an angle $\phi$ between the local directions of the lines of constant $l$ and constant $\alpha$, or equivalently between the lines of constant $b$ and constant $\delta$. With the notation $\mu_{l *} \equiv \mu_{l} \cos b$ and $\mu_{\alpha *} \equiv \mu_{\alpha} \cos \delta$ we write Smart's Eqs. (4), (5) as

$\mu_{l *}=\mu_{\alpha *} \cos \phi+\mu_{\delta} \sin \phi$,

$\mu_{b}=-\mu_{\alpha *} \sin \phi+\mu_{\delta} \cos \phi$.

From spherical trigonometry the angle $\phi$ is given by

$\tan \phi=\frac{\sin \left(\alpha-\alpha_{\mathrm{GP}}\right)}{\cos \delta \tan \delta_{\mathrm{GP}}-\sin \delta \cos \left(\alpha-\alpha_{\mathrm{GP}}\right)}$,

(Smart 1938, Eq. (3)). The angle $\phi$ may also be found by taking the time derivative of the equation defining the transformation equatorial coordinates to Galactic latitude (cf. Lane 1979)

$$
\begin{aligned}
\sin b= & \sin \delta \cos \left(\frac{\pi}{2}-\delta_{\mathrm{GP}}\right) \\
& -\sin \left(\frac{\pi}{2}-\delta_{\mathrm{GP}}\right) \sin \left(\alpha-\alpha_{\mathrm{GP}}-\frac{\pi}{2}\right) \cos \delta,
\end{aligned}
$$

and equating the result to Eq. (A.10).

For Galactic to equatorial we may write analogously to Eqs. (A.9) and (A.10):

$\begin{aligned} \mu_{\alpha *} & =\mu_{l *} \cos \phi_{2}+\mu_{b} \sin \phi_{2}, \\ \mu_{\delta} & =-\mu_{l *} \sin \phi_{2}+\mu_{b} \cos \phi_{2} .\end{aligned}$

We equate the time derivative of Eq. (A.3) to (A.14) to obtain

$\tan \phi_{2}=\frac{-\cos \left(l-l_{\Omega}\right)}{\cot \left(\frac{\pi}{2}-\delta_{\mathrm{GP}}\right) \cos b-\sin \left(l-l_{\Omega}\right) \sin b}$.

Applied to the same source, $\phi=-\phi_{2}$, and thus either angle may be computed with Eq. (A.11) or with Eq. (A.15).

\section{Appendix B: Proper motions and velocity corrections}

The space velocity of a star in the Galaxy may be decomposed into the average space velocity of its surroundings and its velocity with respect to this average, that is its peculiar velocity. The velocity of the local standard of rest for the Sun is its Galactic rotation velocity, $v_{R}\left(R_{0}\right)$, where $R_{0}$ is the distance to the Galactic centre. The peculiar velocity of the Sun is usually written $[U, V, W]$, where the components are respectively in the direction from the Sun towards the Galactic centre, in the direction of the Galactic rotation, and perpendicular to the Galactic plane. The total velocity of the Sun may thus be written

$\boldsymbol{v}_{\odot}=\left[U, V+v_{R}\left(R_{0}\right), W\right]$.

For a pulsar in the Galactic plane, with $b=0$, the velocity of the local standard of rest is also given by the rotation velocity $v_{R}(R)$ around the centre of the galaxy, at the galactocentric distance of the pulsar $R$ (see Fig. B.1). This velocity is in the plane of the galaxy, in the direction perpendicular to the line connection the pulsar to the Galactic center. For a pulsar far from the plane, the meaning of the Local Standard of Rest is less obvious, because the halo stars do not participate in the rotation of the disk. The birthplace of the neutron star is (with the few exceptions mentioned above) in the Galactic plane, therefore we use for its local standard of rest the Galactic rotation $v_{\mathrm{R}}(R)$ of its projection on the Galactic plane. The total velocity of a pulsar at distance $D$ 


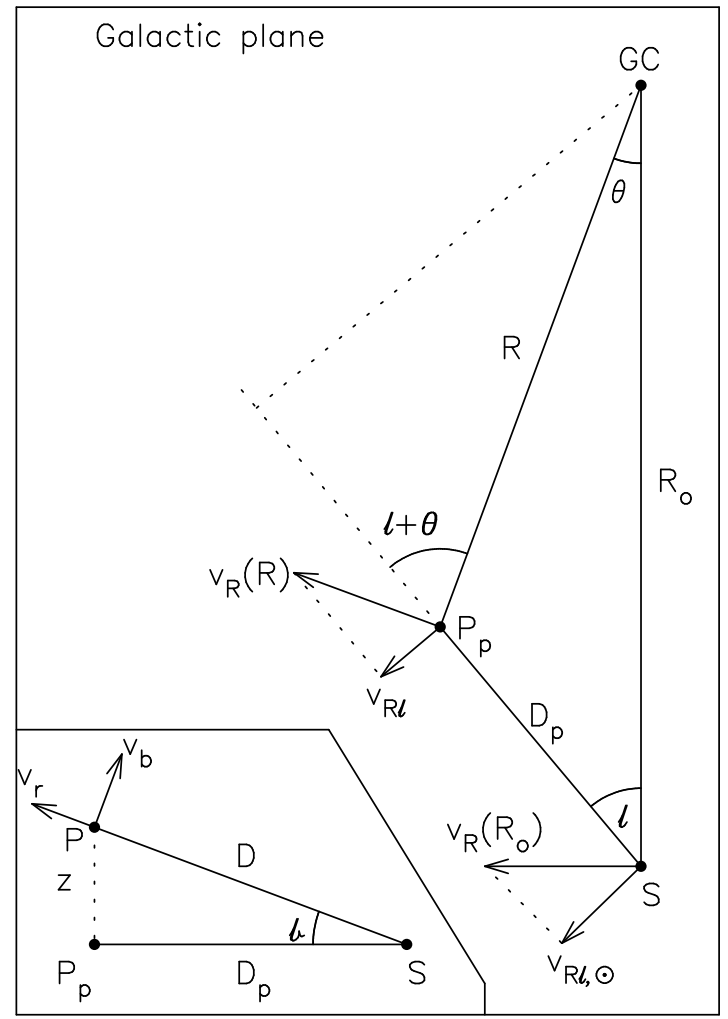

Fig. B.1. Definition of angles and distances in the Galactic plane $(z=0)$, and (inset) of the projected distance $D_{p}$ to the pulsar. $S$ is the Sun, GC the Galactic centre, $P$ the pulsar and $P_{\mathrm{p}}$ the projection of the pulsar position on the Galactic plane.

and Galactic coordinates $l, b$, may be written in the same coordinate frame as used for the Sun (see Fig. B.1):

$\boldsymbol{v}_{p}=\left[U_{p}+v_{R}(R) \sin \theta, V_{p}+v_{R}(R) \cos \theta, W_{p}\right]$,

with $\left[U_{p}, V_{p}, W_{p}\right]$ the peculiar velocity of the pulsar. To obtain the velocity in the $l$-direction, we write the unit vector in this direction as:

$\boldsymbol{l}=[-\sin l, \cos l, 0]$.

Thus the observed relative velocity in the longitude direction is

$\boldsymbol{l} \cdot\left(\boldsymbol{v}_{p}-\boldsymbol{v}_{\odot}\right)=\left(\mu_{l *, v}+\mu_{l *, G}\right) D \equiv \mu_{l *} D$,

where the peculiar velocity in the longitude direction is

$v_{l} \equiv \mu_{l *} D \equiv-U_{p} \sin l+V_{p} \cos l$,

and the correction for Galactic rotation and solar peculiar velocity is

$\mu_{l *, G} D \equiv U \sin l-\left[V+v_{R}\left(R_{0}\right)\right] \cos l+v_{R}(R) \cos (\theta+l)$.

The angle $(\theta+l)$ may be computed from (see Fig. B.1):

$\tan (\theta+l)=\frac{R_{0} \sin l}{R_{0} \cos l-D_{p}}=\frac{R_{0} \sin l}{R_{0} \cos l-D \cos b}$

with $D_{p}$ the projected distance towards the pulsar. Equation (7) follows from Eqs. (B.4)-(B.7).

The unit vector in the $b$-direction may be written

$\boldsymbol{b}=[-\sin b \cos l,-\sin b \sin l, \cos b]$, and the relative velocity in this direction

$\boldsymbol{b} \cdot\left(\boldsymbol{v}_{p}-\boldsymbol{v}_{\odot}\right)=\left(\mu_{b, v}+\mu_{b, G}\right) D \equiv \mu_{b} D$,

with

$v_{b} \equiv \mu_{b, G} D=-U_{p} \sin b \cos l-V_{p} \sin b \sin l+W_{p} \cos b$,

and

$\mu_{b, G} D=U \sin b \cos l+\left[V+v_{R}\left(R_{0}\right)\right] \sin b \sin l-W \cos b$

$-v_{R}(R) \sin (\theta+l) \sin b$.

For a pulsar in direction $l, b$, we can compute $\mu_{l *, G}$ and $\mu_{b, G}$, as a function of distance $D$ from Eqs. (B.6), (B.7) and (B.11). Because the rotation of the sum of two vectors is equal to the sum of two rotated vectors, symbolically: $\mathcal{R}(\boldsymbol{a}+\boldsymbol{\Delta} \boldsymbol{a})=\mathcal{R}(\boldsymbol{a})+\mathcal{R}(\Delta \boldsymbol{a})$, we may rotate the corrections with Eqs. (A.9), (A.10). Hence:

$\mu_{\alpha *, G}=\mu_{l *, G} \cos \phi_{2}+\mu_{b, G} \sin \phi_{2}$,

$\mu_{\delta, G}=-\mu_{l *, G} \sin \phi_{2}+\mu_{b, G} \cos \phi_{2}$,

where $\phi_{2}$ is given by Eq. (A.15).

\section{Appendix C: Numerical evalution of the likelihood in for a semi-anisotropic Maxwellian}

To integrate Eq. (29), we first separate the terms involving the velocity and define

$I_{v}=\mathrm{e}^{-A_{3}} \int_{0}^{\infty} v^{2} \mathrm{e}^{-A_{1} v^{2}-A_{2} v} \mathrm{~d} v, \quad$ where

$A_{1} \equiv \frac{1}{2 \sigma^{2}}+\frac{1}{2}\left(\frac{\sin \xi_{1} \cos \xi_{2}}{D \sigma_{\alpha}}\right)^{2}+\frac{1}{2}\left(\frac{\sin \xi_{1} \sin \xi_{2}}{D \sigma_{\delta}}\right)^{2}$,

$A_{2} \equiv \frac{\sin \xi_{1}}{D}\left[\frac{\left(\mu_{\alpha *, G}-\mu_{\alpha *}^{\prime}\right) \cos \xi_{2}}{\sigma_{\alpha}^{2}}+\frac{\left(\mu_{\delta, G}-\mu_{\delta}^{\prime}\right) \sin \xi_{2}}{\sigma_{\delta}^{2}}\right]$,

$A_{3} \equiv \frac{\left(\mu_{\alpha *, G}-\mu_{\alpha *}^{\prime}\right)^{2}}{2 \sigma_{\alpha}^{2}}+\frac{\left(\mu_{\delta, G}-\mu_{\delta}^{\prime}\right)^{2}}{2 \sigma_{\delta}^{2}}$.

The result of this integral is

$I_{v}=\frac{A_{2} \mathrm{e}^{-A_{3}}}{4 A_{1}^{2}}\left[\sqrt{\pi} \mathrm{e}^{E^{2}}\left(\frac{1}{2 E}+E\right) \operatorname{erfc}(E)-1\right] ; \quad E \equiv \frac{A_{2}}{2 \sqrt{A_{1}}}$.

Entering this in Eq. (29), we obtain:

$$
\begin{aligned}
L_{\text {sim }}(\sigma)= & \int_{0}^{D_{\max }} \int_{0}^{2 \pi} \int_{0}^{\pi} C f_{D}(D) \exp \left[-\frac{\left(1 / D-\varpi^{\prime}\right)^{2}}{2 \sigma_{\varpi}^{2}}\right] \\
& \times \sin \xi_{1} 2 \sqrt{\frac{2}{\pi}} \frac{1}{\sigma^{3}} I_{v}\left(D, \xi_{1}, \xi_{2}\right) \mathrm{d} \xi_{1} \mathrm{~d} \xi_{2} \mathrm{~d} D .
\end{aligned}
$$

Returning to Eq. (28), we note that for fixed distance $D$, velocity $v$ and angle $\xi_{1}, P_{\text {sim }}$ reaches it maximum when the arguments of the exponents that include the proper motions are zero. The value of $\xi_{2}$ for which this is the case follows from

$\tan \xi_{2 m}=\frac{\mu_{\alpha *}^{\prime}-\mu_{\alpha * G}(D)}{\mu_{\delta}^{\prime}-\mu_{\delta, G}(D)}$.

Because this angle is the same for every $v$, the same value of $\xi_{2}$ also maximizes the integrand of Eq. (C.3). The integration of Eq. (C.3) is done in three steps. First we fix 
$D$ and $\xi_{1}$, and determine the range of $\xi_{2}$ from the condition Eq. (27) (or equivalently by testing with Eq. (26) that $v_{z}$ is in the right direction). We divide this range in three parts, one given by $\left(\xi_{2 m}-h\right)$ to $\left(\xi_{2 m}+h\right)$, and the other two dividing the remaining range, and integrate over $\xi_{2}$ in each part separately with a 64-node Gaussian quadrature.
We find that $h=2 \pi / 70$ leads to accurate results. Second, we integrate over $\xi_{1}$ with one 64-node Gaussian quadrature. Finally, we integrate over $D$, in steps of $100 \mathrm{pc}$, for $D_{\max }=10 \mathrm{kpc}$.

We compute $L_{\text {maxw-si }}(\sigma)$ on a grid of values of $\sigma$, in steps of $5 \mathrm{~km} \mathrm{~s}^{-1}$, interpolate linearly to get a grid with steps of $1 \mathrm{~km} \mathrm{~s}^{-1}$.

\section{Appendix D: Master list}

Table D.1. Master list of the pulsars used in our study.

\begin{tabular}{|c|c|c|c|c|c|c|c|c|c|c|c|c|c|}
\hline & B-name & $\mathrm{J}$-name & & $\begin{array}{l}l \\
\left(^{\circ}\right)\end{array}$ & $\begin{array}{l}b \\
\left({ }^{\circ}\right)\end{array}$ & $\begin{array}{l}\varpi^{\prime} \\
(\mathrm{m}\end{array}$ & $\sigma_{\varpi}$ & $\begin{array}{c}\mu_{\alpha *}^{\prime} \\
\text { (mas }\end{array}$ & $\sigma_{\alpha}$ & $\begin{array}{c}\mu_{\delta}^{\prime} \\
(\mathrm{ma}\end{array}$ & $\begin{array}{l}\sigma_{\delta} \\
/ \mathrm{yr})\end{array}$ & $\begin{array}{c}\tau_{\mathrm{c}} \\
(\mathrm{Myr})\end{array}$ & Ref. \\
\hline 1 & & J0034-0721 & & 110.42 & -69.82 & 0.93 & 0.08 & 10.37 & 0.08 & -11.13 & 0.16 & 36.7 & 6 \\
\hline 2 & & J0108-1431 & & 140.93 & -76.82 & 4.17 & 1.42 & 75.05 & 2.26 & -152.54 & 1.65 & 166.4 & 5 \\
\hline 3 & B $0136+57$ & $\mathrm{~J} 0139+5814$ & & 129.22 & -4.04 & 0.37 & 0.04 & -19.11 & 0.07 & -16.60 & 0.07 & 0.4 & 6 \\
\hline 4 & В0329+54 & $\mathrm{J} 0332+5434$ & $\mathrm{i}$ & 145.00 & -1.22 & 0.94 & 0.11 & 17.00 & 0.27 & -9.48 & 0.37 & 5.5 & 1 \\
\hline 5 & B $0355+54$ & $\mathrm{~J} 0358+5413$ & $\mathrm{i}$ & 148.19 & 0.81 & 0.91 & 0.16 & 9.20 & 0.18 & 8.17 & 0.39 & 0.6 & 4 \\
\hline 6 & B $0450+55$ & $\mathrm{~J} 0454+5543$ & & 152.62 & 7.55 & 0.84 & 0.05 & 53.34 & 0.06 & -17.56 & 0.14 & 2.3 & 6 \\
\hline 7 & & $\mathrm{~J} 0538+2817$ & $\mathrm{i}$ & 179.72 & -1.69 & 0.72 & 0.12 & -23.57 & 0.10 & 52.87 & 0.10 & 0.6 & 6 \\
\hline 8 & B0628-28 & J0630-2834 & & 236.95 & -16.76 & 3.01 & 0.41 & -46.30 & 0.99 & 21.26 & 0.52 & 2.8 & 5 \\
\hline 9 & B0656+14 & $\mathrm{J} 0659+1414$ & $\mathrm{i}$ & 201.11 & 8.26 & 3.47 & 0.36 & 44.07 & 0.63 & -2.40 & 0.29 & 0.1 & 2 \\
\hline 10 & B $0809+74$ & J0814+7429 & & 140.00 & 31.62 & 2.31 & 0.04 & 24.02 & 0.09 & -43.96 & 0.35 & 122.0 & 1 \\
\hline 11 & B0818-13 & J0820-1350 & & 235.89 & 12.59 & 0.51 & 0.04 & 21.64 & 0.09 & -39.44 & 0.05 & 9.3 & 6 \\
\hline 12 & B0919+06 & $\mathrm{J} 0922+0638$ & & 225.42 & 36.39 & 0.83 & 0.13 & 18.35 & 0.06 & 86.56 & 0.12 & 0.5 & 3 \\
\hline 13 & В0950+08 & $\mathrm{J} 0953+0755$ & & 228.91 & 43.70 & 3.82 & 0.07 & -2.09 & 0.08 & 29.46 & 0.07 & 17.5 & 1 \\
\hline 14 & B1133+16 & $\mathrm{J} 1136+1551$ & & 241.90 & 69.20 & 2.80 & 0.16 & -73.95 & 0.38 & 368.05 & 0.28 & 5.0 & 1 \\
\hline 15 & B1237+25 & $\mathrm{J} 1239+2453$ & & 252.45 & 86.54 & 1.16 & 0.08 & -106.82 & 0.17 & 49.92 & 0.18 & 22.9 & 1 \\
\hline 16 & B $1508+55$ & $\mathrm{~J} 1509+5531$ & & 91.33 & 52.29 & 0.47 & 0.03 & -73.64 & 0.05 & -62.65 & 0.09 & 2.3 & 6 \\
\hline 17 & B $1541+09$ & $\mathrm{~J} 1543+0929$ & & 17.81 & 45.78 & 0.13 & 0.02 & -7.61 & 0.06 & -2.87 & 0.07 & 27.5 & 6 \\
\hline 18 & B1556-44 & $\mathrm{J} 1559-4438$ & & 334.54 & 6.37 & 0.38 & 0.08 & 1.52 & 0.14 & 13.15 & 0.05 & 4.0 & 5 \\
\hline 19 & B1929+10 & $\mathrm{J} 1932+1059$ & $\mathrm{i}$ & 47.38 & -3.88 & 2.78 & 0.06 & 94.06 & 0.09 & 43.24 & 0.17 & 3.1 & 7 \\
\hline 20 & & $\mathrm{~J} 1935+1616$ & & 52.44 & -2.09 & 0.22 & 0.12 & 1.13 & 0.13 & -16.09 & 0.15 & 0.9 & 6 \\
\hline 21 & B2016+28 & $\mathrm{J} 2018+2839$ & & 68.10 & -3.98 & 1.03 & 0.10 & -2.64 & 0.21 & -6.17 & 0.38 & 59.8 & 1 \\
\hline 22 & B2020+28 & $\mathrm{J} 2022+2854$ & & 68.86 & -4.67 & 0.61 & 0.08 & -3.46 & 0.17 & -23.73 & 0.21 & 2.9 & 7 \\
\hline 23 & $\mathrm{~B} 2021+51$ & $\mathrm{~J} 2022+5154$ & & 87.86 & 8.38 & 0.78 & 0.07 & -5.03 & 0.27 & 10.96 & 0.17 & 2.7 & 7 \\
\hline 24 & B2045-16 & J2048-1616 & & 30.51 & -33.08 & 1.05 & 0.03 & 113.16 & 0.02 & -4.60 & 0.28 & 2.8 & 6 \\
\hline 25 & B2053+36 & $\mathrm{J} 2055+3630$ & & 79.13 & -5.59 & 0.17 & 0.03 & 1.04 & 0.04 & -2.46 & 0.13 & 9.5 & 6 \\
\hline 26 & & $\mathrm{~J} 2144-3933$ & $\mathrm{i}$ & 2.79 & -49.47 & 6.05 & 0.56 & -57.89 & 0.88 & -155.90 & 0.54 & 272.3 & 5 \\
\hline 27 & B2154+40 & $\mathrm{J} 2157+4017$ & & 90.49 & -11.34 & 0.28 & 0.06 & 16.13 & 0.10 & 4.12 & 0.12 & 7.1 & 6 \\
\hline 28 & B $2310+42$ & $\mathrm{~J} 2313+4253$ & & 104.41 & -16.42 & 0.93 & 0.07 & 24.15 & 0.10 & 5.95 & 0.13 & 49.3 & 6 \\
\hline
\end{tabular}

Notes. The last column gives the reference in Table 2 from which the parallax with error (Cols. 7, 8), and the proper motions with their errors (Cols. 9-12) are taken. In the case of asymmetric errors we take the larger one. Columns 11 gives the characteristic age $\tau_{\mathrm{c}} \equiv P /(2 \dot{P})$. An i in Col. 5 indicates that the model velocity distribution for this pulsar is isotropic in the models that mix isotropic and semi-isotropic velocity distributions. 